\title{
Why are ELEvoHI CME arrival predictions different if based on STEREO-A or STEREO-B heliospheric imager observations?
}

Article

Published Version

Creative Commons: Attribution 4.0 (CC-BY)

Open acces

Hinterreiter, J. ORCID: https://orcid.org/0000-0002-1222-8243, Amerstorfer, T. ORCID: https://orcid.org/0000-0001-90246706, Reiss, M. A., Möstl, C. ORCID: https://orcid.org/00000001-6868-4152, Temmer, M. ORCID: https://orcid.org/00000003-4867-7558, Bauer, M., Amerstorfer, U. V. ORCID: https://orcid.org/0000-0003-1516-5441, Bailey, R. L. ORCID: https://orcid.org/0000-0003-2021-6557, Weiss, A. J., Davies, J. A., Barnard, L. A. ORCID: https://orcid.org/0000-0001-98764612 and Owens, M. J. ORCID: https://orcid.org/0000-00032061-2453 (2021) Why are ELEvoHI CME arrival predictions different if based on STEREO-A or STEREO-B heliospheric imager observations? Space Weather, 19 (3). e2020SW002674. ISSN 1542-7390 doi: https://doi.org/10.1029/2020SW002674 Available at https://centaur.reading.ac.uk/96267/ 
Publisher: AGU

All outputs in CentAUR are protected by Intellectual Property Rights law, including copyright law. Copyright and IPR is retained by the creators or other copyright holders. Terms and conditions for use of this material are defined in the End User Agreement.

\section{www.reading.ac.uk/centaur}

\section{CentAUR}

Central Archive at the University of Reading

Reading's research outputs online 


\section{Space Weather}

\section{RESEARCH ARTICLE \\ 10.1029/2020SW002674 \\ Special Section: \\ Heliophysics and Space Weather \\ Studies from the Sun-Earth \\ Lagrange Points \\ Key Points: \\ - A comparison of CME arrival time and speed predictions from two vantage points was carried out using ELEvoHI \\ - A highly structured ambient solar wind flow leads to larger arrival time differences between STA and STB predictions \\ - The assumption of a rigid CME front in ELEvoHI and other HI- based methods is most probably too simplistic}

Correspondence to:

J. Hinterreiter,

juergen.hinterreiter@oeaw.ac.at

\section{Citation:}

Hinterreiter, J., Amerstorfer, T., Reiss, M. A., Möstl, C., Temmer, M., Bauer, M., et al. (2021). Why are ELEvoHI CME arrival predictions different if based on STEREO-A or STEREO-B Heliospheric Imager observations? Space Weather, 19, e2020SW002674. https://doi.org/10.1029/2020SW002674

Received 30 OCT 2020 Accepted 29 JAN 2021
(C) 2021. The Authors.

This is an open access article under the terms of the Creative Commons Attribution License, which permits use, distribution and reproduction in any medium, provided the original work is properly cited.

\section{Why are ELEvoHI CME Arrival Predictions Different if Based on STEREO-A or STEREO-B Heliospheric Imager Observations?}

\author{
Jürgen Hinterreiter ${ }^{1,2}$ (D), Tanja Amerstorfer ${ }^{1}$ (D), Martin A. Reiss ${ }^{1,3}$ (D), \\ Christian Möstl $^{1,3}$ (D), Manuela Temmer ${ }^{2}$ (D), Maike Bauer ${ }^{1,2}$ (D), Ute V. Amerstorfer ${ }^{1}$ (D),

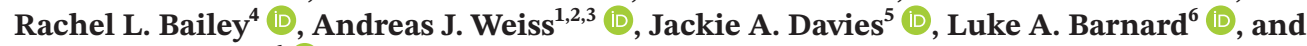 \\ Mathew J. Owens ${ }^{6}$ (D) \\ ${ }^{1}$ Space Research Institute, Austrian Academy of Sciences, Graz, Austria, ${ }^{2}$ Institute of Physics, University of Graz, \\ Graz, Austria, ${ }^{3}$ Institute of Geodesy, Graz University of Technology, Graz, Austria, ${ }^{4}$ Conrad Observatory, Zentralanstalt \\ für Meteorologie und Geodynamik, Vienna, Austria, ${ }^{5}$ RAL Space, Rutherford Appleton Laboratory, Didcot, UK, \\ ${ }^{6}$ Department of Meteorology, University of Reading, Reading, UK
}

Abstract Accurate forecasting of the arrival time and arrival speed of coronal mass ejections (CMEs) is an unsolved problem in space weather research. In this study, a comparison of the predicted arrival times and speeds for each CME based, independently, on the inputs from the two STEREO vantage points is carried out. We perform hindcasts using ELlipse Evolution model based on Heliospheric Imager observations (ELEvoHI) ensemble modeling. An estimate of the ambient solar wind conditions is obtained by the Wang-Sheeley-Arge/Heliospheric Upwind eXtrapolation (WSA/HUX) model combination that serves as input to ELEvoHI. We carefully select 12 CMEs between February 2010 and July 2012 that show clear signatures in both STEREO-A and STEREO-B HI time-elongation maps, that propagate close to the ecliptic plane, and that have corresponding in situ signatures at Earth. We find a mean arrival time difference of $6.5 \mathrm{~h}$ between predictions from the two different viewpoints, which can reach up to $9.5 \mathrm{~h}$ for individual CMEs, while the mean arrival speed difference is $63 \mathrm{~km} \mathrm{~s}^{-1}$. An ambient solar wind with a large speed variance leads to larger differences in the STEREO-A and STEREO-B CME arrival time predictions $(c c=0.92)$. Additionally, we compare the predicted arrivals, from both spacecraft, to the actual in situ arrivals at Earth and find a mean absolute error of $7.5 \pm 9.5 \mathrm{~h}$ for the arrival time and $87 \pm 111 \mathrm{~km} \mathrm{~s}^{-1}$ for the arrival speed. There is no tendency for one spacecraft to provide more accurate arrival predictions than the other.

\section{Introduction}

Understanding the dynamics of coronal mass ejections (CMEs) in the heliosphere is a key aspect of space weather research. CMEs are huge clouds of energetic and magnetized plasma (Hundhausen et al., 1994) erupting from the solar corona that may reach speeds of up to $3,000 \mathrm{~km} \mathrm{~s}^{-1}$. When they hit Earth, CMEs can produce strong geomagnetic storms (Gosling et al., 1990; Kilpua et al., 2012; Richardson \& Cane, 2012; Srivastava \& Venkatakrishnan, 2004; Venkatakrishnan, 2004) causing communication and navigation system problems, damaging satellites and can even cause power outages (Cannon, 2013). The need for accurate predictions of CMEs, both CME arrival time and speed, is becoming increasingly important (Owens et al., 2020), because humankind, more than ever, depends on advanced technology.

Shortly after their eruption, CMEs can be observed in coronagraph images. Two of the few space-borne coronagraphs in operation are the Large Angle and Spectrometric Coronagraph (LASCO) C2 and C3 onboard the Solar and Heliospheric Observatory (SoHO; Brueckner et al., 1995). SoHO is situated in a Lissajous orbit around Lagrange point 1 (L1), about 1.5 million km upstream of Earth in the Sun-Earth line.

The launch of the Solar Terrestrial Relations Observatory (STEREO; Kaiser et al., 2008) twin-spacecraft mission in 2006 provided an unprecedented opportunity to observe CMEs from off the Sun-Earth line. The two spacecraft orbit the Sun slightly closer (STEREO Ahead; STA) and slightly further (STEREO Behind; STB) than Earth, leading to a separation of each spacecraft by about $22^{\circ}$ per year from Earth in opposite directions. Both spacecraft are equipped with the in situ Measurements of Particles and CME Transients 
(IMPACT; Luhmann et al., 2008) instrument package to measure solar wind speed, density and magnetic field and additionally host a suite of imagers, such as the COR1 and COR2 (Howard et al., 2008) coronagraphs and the heliospheric imagers, HI1 and HI2 (Eyles et al., 2009). The wide-angle HI cameras provide observations of the heliosphere that allow us to track a CME from close to the Sun out to the orbit of Earth, particularly in the ecliptic plane.

CMEs are optically thin structures that expand rapidly, and decreasing density lowers the line-of-sight integrated intensity in white-light data. As a consequence, the tracking of CME fronts and the interpretation of HI image data is difficult. Furthermore, the plane-of-sky assumption is not valid, and we must assume a certain longitudinal extent of the CME frontal shape.

CMEs may be influenced by different phenomena in the heliosphere, e.g., magnetic forces close to the Sun, high-speed solar wind streams, or by other CMEs (Kay \& Opher, 2015; Lugaz et al., 2012; Möstl et al., 2015). The ambient solar wind can also affect the kinematic and morphological characteristics of CMEs (e.g., Gopalswamy et al., 2000; Gosling et al., 1990; Manoharan et al., 2004). A CME originating at a speed much faster than the ambient solar wind speed is likely to experience deceleration while slow CMEs may accelerate during their propagation (Manoharan \& Mujiber Rahman, 2011; Richardson \& Cane, 2010). Hence, not only the propagation direction but also the kinematics and shape of CMEs can be altered (e.g., Kay \& Nieves-Chinchilla, 2020; Liu et al., 2014; Rollett et al., 2014; Ruffenach et al., 2015; Savani et al., 2010; Zuccarello et al., 2012). By tracking CMEs far out in the heliosphere, we get an understanding of their interaction with the ambient solar wind and corotating interaction regions.

Over the last decades, a vast number of CME prediction models have been developed. They include empirical models, for example, Effective Acceleration Model (EAM; Paouris \& Mavromichalaki, 2017), which use relationships between observable parameters and the transit time. There are also drag-based models (e.g., DBM; Vršnak et al., 2013, DBEM; Dumbović et al., 2018, ANTEATR; Kay et al., 2020), that make use of physics-based equations and account for drag between the ambient solar wind and the CME. Other models make use of $\mathrm{HI}$ images, which require techniques to convert the measured elongation into radial distance. For example, the fixed phi fitting (FPF; Rouillard et al., 2008; Sheeley et al., 1999) technique considers a CME as a single point, propagating at a constant speed, and provides an estimate of the constant direction of the CME propagation relative to the observer from the apparent acceleration within a sequence of $\mathrm{HI}$ images. The harmonic mean fitting (HMF; Lugaz, 2010; Möstl et al., 2011) method is similar except that it describes a CME as a circle that remains attached to the Sun center. The self-similar-expansion fitting (SSEF; Davies et al., 2012; Lugaz et al., 2010; Möstl \& Davies, 2013) technique describes a CME as a circle having an increasing radius as it propagates away from the Sun in such a way that it maintains a constant angular width. FPF and HMF are extremes of the SSEF technique with a half width of $0^{\circ}$ and $90^{\circ}$, respectively. More sophisticated models combine both the drag-based approach and HI observations (e.g., DBM fitting; Žic et al., 2015, ELlipse Evolution model based on HI observations, ELEvoHI; Amerstorfer et al., 2018; Rollett et al., 2016). Finally, numerical models, which are computational heavy, solve magnetohydrodynamic (MHD) equations (e.g., ENLIL; Odstrcil et al., 2004, EUHFORIA; Pomoell \& Poedts, 2018) simulating the ambient solar wind in the full heliosphere based on synoptic photospheric magnetic-field maps. CMEs are then injected into these models to provide predictions regarding the arrival time and arrival speed at different locations in the heliosphere.

As noted above, ELEvoHI aims to predict the arrival time and arrival speed of CMEs. The model assumes an elliptical shape for the CME front and incorporates the drag exerted by the ambient solar wind. Also, different sources of ambient solar wind speed (e.g., provided by numerical models) can serve as input to ELEvoHI (Amerstorfer et al., 2020). In its latest version, the model can be used with STEREO-A HI beacon mode data to provide near real-time CME arrival predictions.

This study assesses ELEvoHI to evaluate arrival time and speed predictions of past CMEs using STEREO HI science-quality data. We perform ELEvoHI ensemble predictions for 12 CMEs, where each CME is modeled using input data from STA and STB, separately. In an idealized case, in which a CME with an elliptical front propagates in an ambient solar wind that is constant in space and time, one would expect to get similar results for the arrival time and arrival speed from the two different vantage points. Instead of inferring the propagation directions of the events under study from HI images (e.g., FPF, SSEF), as was 
done by Amerstorfer et al. (2018), we make use of coronagraph images and perform Graduated Cylindrical Shell (GCS; Thernisien et al., 2006, 2009) reconstruction for each CME based on multivantage point coronagraph data. Additionally, we apply a combination of the Wang-Sheeley-Arge (Arge et al., 2003) and the Heliospheric Upwind eXtrapolation (Owens \& Riley, 2017; Riley \& Lionello, 2011) model (WSA/HUX model combination; Reiss et al., 2019, 2020) to get an estimate of the ambient solar wind conditions in the heliosphere through which the CME propagates. With the additional information about the propagation direction of the CME and the modeled ambient solar wind, ELEvoHI is more likely to give better arrival time and arrival speed predictions.

In Section 2, we describe our data selection process, including the data products, and list all of the studied CMEs. Section 3 deals with the ELEvoHI setup and how the input data required by the model is obtained. In Section 4 , we present our results and give reasons for the difference in the model predictions based on STA and STB input data. The discussion and further implementations of the model are included in Section 5.

\section{Data Preparation}

We select a period between February 2010 and July 2012 during which the STEREO spacecraft had a separation angle from Earth of about $65^{\circ}$ to $120^{\circ}$, respectively, from which we study 12 CMEs. The HELCATS HICAT CME catalog lists about 700 entries over this time range (Harrison et al., 2018). However, our list is constrained to 12 events, since the CMEs have to:

(1) be observed by HI on both STA and STB spacecraft (as listed in the HIJoinCAT; Barnes et al., 2020)

(2) propagate close to the ecliptic plane

(3) have a corresponding in situ signature at Earth

(4) be able to be tracked unambiguously in time-elongation maps

Table 1 contains the list of selected CMEs with their unique identifier and the time of their first observation in HI1 images (according to the HELCATS catalog Version 6). The interplanetary CME (ICME) times and speeds are taken from version 2.0 of the HELCATS ICMECAT catalog (Möstl et al., 2020, see also the links in the data section). The ICMECAT assimilates ICME catalogs from different spacecraft into one consistent list, and was first published in Möstl et al. (2017). The ICME date as observed by the Wind spacecraft is defined by the shock arrival time, or, if no shock is present, the start of a density enhancement in front of the magnetic flux rope (MFR). If neither is observed, the ICME start time is taken as the start time of the MFR. The corresponding ICME speed is the mean proton bulk speed of either the sheath region, the density enhancement ahead of the MFR, or the speed of the MFR itself. The spread in the speed over the given interval for each event is indicated in Table 1 by a standard deviation. For Table 1, some times in the ICMECAT were originally taken from the Wind ICME catalog (Nieves-Chinchilla et al., 2018), while other events that were not present in the Wind catalog were added by Möstl et al. (2020) to the HELCATS ICMECAT.

To run ELEvoHI, we make use of several data products. Most important are images from HI onboard STEREO. The HI instrument on each STEREO spacecraft consists of two white-light wide-angle imagers, HI1 and HI2. HI1 has a field-of-view (FOV) extending from $4^{\circ}$ to $24^{\circ}$ elongation (angle from Sun center) in the ecliptic and HI2 has an angular FOV extending from $18.8^{\circ}$ to $88.8^{\circ}$ elongation in the ecliptic. The nominal cadence of the HI1 and HI2 science data is 40 and $120 \mathrm{~min}$, respectively. The science image bin size is 70 arc sec for HI1 and 4 arc min for HI2. For the additional input parameters to ELEvoHI, we developed the Ecliptic cut Angles from GCS for ELEvoHI tool (EAGEL, see Section 3.1). EAGEL ideally uses coronagraph images from STEREO COR1/COR2 and from LASCO C2/C3 onboard SoHO, but images from at least two different viewpoints are required. The FOV of COR1 ranges from 1.4 to $4 R_{\odot}$ and COR2, from 2 to $15 R_{\odot}$, while $\mathrm{C} 2$ has an FOV of 1.5-6 $R_{\odot}$ and $\mathrm{C} 3,3.7-30 R_{\odot}$ (all quoted in the plane-of-sky). The cadence of the coronagraph science images is about $15 \mathrm{~min}$.

\section{Methods}

\subsection{EAGEL (Ecliptic Cut Angles From GCS for ELEvoHI)}

In this section, we present a newly developed Interactive Data Language (IDL ${ }^{\mathrm{TM}}$ ) tool called EAGEL (Ecliptic cut Angles from GCS for ELEvoHI). EAGEL allows any user to determine the propagation direction, $\phi$, 
Table 1

List of Selected CMEs

\begin{tabular}{|c|c|c|c|c|c|c|c|}
\hline No. & ID STA & Date STA & ID STB & Date STB & ICMECAT ID & ICME date & $\begin{array}{c}v_{\mathrm{ICME}} \\
\left(\mathrm{km} \mathrm{s}^{-1)}\right.\end{array}$ \\
\hline 1 & HCME_A_20100203_01 & $\begin{array}{l}\text { February 03, } \\
201014: 49\end{array}$ & HCME_B_20100203_01 & $\begin{array}{l}\text { February 03, } \\
201020: 49\end{array}$ & $\begin{array}{l}\text { ICME_Wind_ } \\
\text { NASA_20100207_01 }\end{array}$ & $\begin{array}{l}\text { February } 07 \\
\quad 201018: 04^{\mathrm{b}}\end{array}$ & $406 \pm 2$ \\
\hline 2 & HCME_A_20100319_01 & $\begin{array}{l}\text { March 19, } 2010 \\
\quad 22: 09\end{array}$ & HCME_B_20100319_01 & $\begin{array}{l}\text { March 19, } 2010 \\
20: 09\end{array}$ & $\begin{array}{l}\text { ICME_Wind_ } \\
\quad \text { MOESTL_20100323_01 }\end{array}$ & $\begin{array}{l}\text { March } 23,2010 \\
22: 29^{\mathrm{c}}\end{array}$ & $292 \pm 12$ \\
\hline 3 & HCME_A_20100403_01 & $\begin{array}{l}\text { April 03, } 2010 \\
\text { 12:09 }\end{array}$ & HCME_B_20100403_01 & $\begin{array}{l}\text { April 03, } 2010 \\
\text { 12:09 }\end{array}$ & $\begin{array}{l}\text { ICME_Wind_ } \\
\text { NASA_20100405_01 }\end{array}$ & $\begin{array}{c}\text { April 05, } 2010 \\
07: 55^{\mathrm{a}}\end{array}$ & $734 \pm 18$ \\
\hline 4 & HCME_A_20100408_01 & $\begin{array}{c}\text { April 08, } 2010 \\
\text { 06:49 }\end{array}$ & HCME_B_20100408_01 & $\begin{array}{c}\text { April 08, } 2010 \\
07: 29\end{array}$ & $\begin{array}{l}\text { ICME_Wind_ } \\
\text { NASA_20100411_01 }\end{array}$ & $\begin{array}{c}\text { April 11, } 2010 \\
12: 20^{\mathrm{a}}\end{array}$ & $432 \pm 17$ \\
\hline 5 & HCME_A_20100523_01 & $\begin{array}{c}\text { May } 23,2010 \\
22: 09\end{array}$ & HCME_B_20100524_01 & $\begin{array}{c}\text { May } 24,2010 \\
\text { 00:09 }\end{array}$ & $\begin{array}{l}\text { ICME_Wind_ } \\
\text { NASA_20100528_01 }\end{array}$ & $\begin{array}{c}\text { May } 28,2010 \\
01: 52^{\mathrm{a}}\end{array}$ & $370 \pm 10$ \\
\hline 6 & HCME_A_20101026_01 & $\begin{array}{l}\text { October } 26, \\
201015: 29\end{array}$ & HCME_B_20101026_01 & $\begin{array}{l}\text { October } 26 \\
201016: 10\end{array}$ & $\begin{array}{l}\text { ICME_Wind_ } \\
\quad \text { MOESTL_20101030_01 }\end{array}$ & $\begin{array}{l}\text { October 30, } \\
\quad 201009: 15^{\mathrm{b}}\end{array}$ & $380 \pm 9$ \\
\hline 7 & HCME_A_20110130_01 & $\begin{array}{l}\text { January 30, } \\
201120: 09\end{array}$ & HCME_B_20110130_01 & $\begin{array}{l}\text { January 30, } \\
2011 \text { 18:49 }\end{array}$ & $\begin{array}{l}\text { ICME_Wind_ } \\
\quad \text { MOESTL_20110204_01 }\end{array}$ & $\begin{array}{l}\text { February 04, } \\
201101: 50^{\mathrm{a}}\end{array}$ & $375 \pm 9$ \\
\hline 8 & HCME_A_20110214_02 & $\begin{array}{l}\text { February 14, } \\
201122: 49\end{array}$ & HCME_B_20110214_02 & $\begin{array}{l}\text { February 14, } \\
201122: 09\end{array}$ & $\begin{array}{l}\text { ICME_Wind_ } \\
\quad \text { MOESTL_20110218_01 }\end{array}$ & $\begin{array}{l}\text { February 18, } \\
201100: 48^{\mathrm{a}}\end{array}$ & $493 \pm 25$ \\
\hline 9 & HCME_A_20110906_02 & $\begin{array}{r}\text { September 06, } \\
201123: 29\end{array}$ & HCME_B_20110907_01 & $\begin{array}{r}\text { September 07, } \\
2011 \text { 03:29 }\end{array}$ & $\begin{array}{l}\text { ICME_Wind_ } \\
\quad \text { MOESTL_20110909_01 }\end{array}$ & $\begin{array}{l}\text { September 09, } \\
201111: 46^{\mathrm{a}}\end{array}$ & $417 \pm 20$ \\
\hline 10 & HCME_A_20120123_01 & $\begin{array}{l}\text { January } 23, \\
2012 \text { 04:49 }\end{array}$ & HCME_B_20120123_01 & $\begin{array}{l}\text { January 23, } \\
2012 \text { 05:29 }\end{array}$ & $\begin{array}{l}\text { ICME_Wind_ } \\
\text { MOESTL_20120124_01 }\end{array}$ & $\begin{array}{l}\text { January } 24, \\
201214: 36^{\mathrm{a}}\end{array}$ & $613 \pm 36$ \\
\hline 11 & HCME_A_20120614_01 & $\begin{array}{c}\text { June } 14,2012 \\
16: 09\end{array}$ & HCME_B_20120614_01 & $\begin{array}{c}\text { June 14, } 2012 \\
16: 09\end{array}$ & $\begin{array}{l}\text { ICME_Wind_ } \\
\text { MOESTL_20120616_01 }\end{array}$ & $\begin{array}{c}\text { June } 16,2012 \\
19: 34^{\mathrm{a}}\end{array}$ & $489 \pm 29$ \\
\hline 12 & HCME_A_20120712_02 & $\begin{array}{c}\text { July } 12,2012 \\
18: 49\end{array}$ & HCME_B_20120712_01 & $\begin{array}{c}\text { July 12, } 2012 \\
18: 09\end{array}$ & $\begin{array}{l}\text { ICME_Wind_ } \\
\text { MOESTL_20120714_01 }\end{array}$ & $\begin{array}{c}\text { July } 14,2012 \\
17: 38^{\mathrm{a}}\end{array}$ & $615 \pm 37$ \\
\hline
\end{tabular}

Note. ID and Date correspond to the unique identifier and the time of the first appearance of the CME in HI1 imagery, from the HELCATS catalog, for STA and STB spacecraft. ICMECAT ID is the identifier of the interplanetary coronal mass ejection (ICME) from an updated version of the HELCATS ICMECAT (Möstl et al., 2017), ICME date is the start time of the detected ICME and $v_{\text {ICME }}$ is the measured in situ arrival speed obtained from the HELCATS ICMECAT.

${ }^{a}$ Shock arrival time. ${ }^{\mathrm{b}}$ Time of density enhancement. ${ }^{\mathrm{c}}$ Time of the magnetic flux rope.

and the half width, $\lambda$, within the ecliptic plane, based on GCS reconstruction of a CME. To perform GCS reconstruction, coronagraph images from at least two vantage points (STEREO and/or LASCO) are required. EAGEL provides the routines to download the required coronagraph images, combines all the functions to perform GCS reconstruction, and produces a cut in the ecliptic plane. Standard preprocessing of the images is implemented in EAGEL to make the CME features clearly visible to the user, who can decide between using background-subtracted, running-difference, and base-difference images. The user can then perform GCS reconstruction using the IDL SolarSoft procedure rtsccguicloud. The top row of Figure 1a shows the coronagraph images (from left to right: STB/COR2, LASCO/C2, STA/COR2) for event \#5. The bottom row additionally shows the GCS wire frame (green mesh). In its current version, ELEvoHI is a 2D prediction model giving results only in the ecliptic plane. Therefore, EAGEL calculates the ecliptic part of the GCS wire frame and selects the boundaries of the ecliptic cut (see red and green line in Figure 1b). The boundaries are defined to be the outermost points of each side of the ecliptic cut with respect to the apex direction from GCS reconstruction. This gives $\lambda$ and $\phi$, where the latter is defined to be exactly in between the two boundaries. A plot is shown to the user (Figure 1b) and, if needed, the boundaries can be changed manually. Once the user approves the selection, $\lambda$ and $\phi$ relative to Earth and to the two STEREO spacecraft are stored and can be used by ELEvoHI.

In Table 2, we list the time (Date) of the STEREO coronagraph images used to get $\lambda$ and $\phi$ for each event. EAGEL then selects the SoHO coronagraph images closest in time to the quoted date. Each CME is fitted once based on the three different viewpoints (STA, STB, LASCO). However, for event \#1 no LASCO data are available, so GCS reconstruction is based on STEREO images only. For all the events, the times of the 
(a)

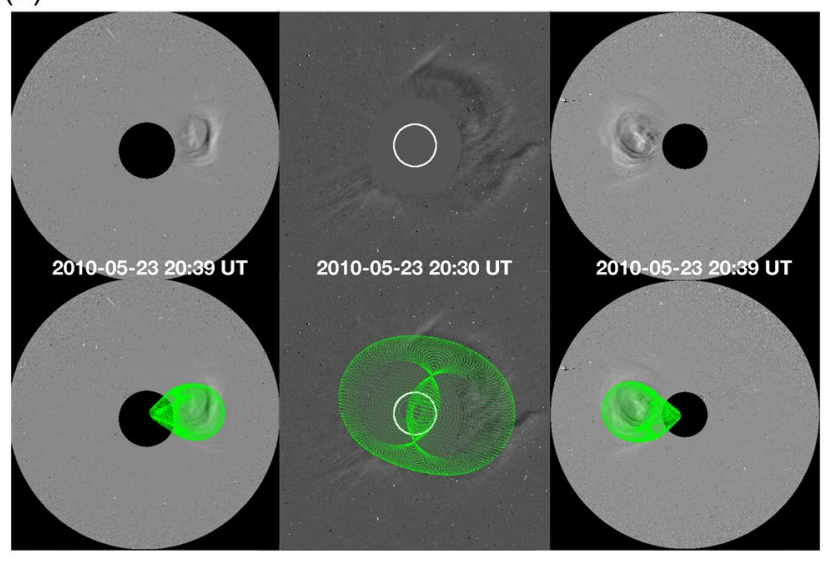

(b)

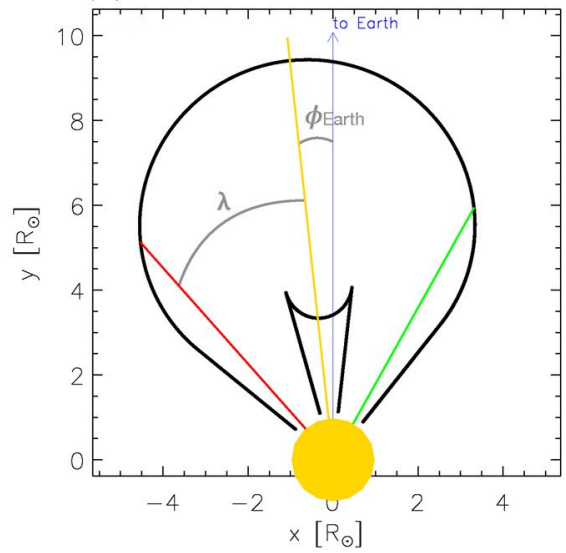

Figure 1. GCS reconstruction (left) and ecliptic cut of the wire frame (right) for event \#5. (a) Top row from left to right: STB/COR2, LASCO/C2, STA/COR2. Bottom row: same as top row but with the GCS wire frame overlaid. (b) Ecliptic cut (black) of the GCS wire frame. Red and green lines show the boundaries selected by either EAGEL or the user. The yellow line defines the ecliptic propagation direction with respect to Earth, $\phi_{\text {Earth }}$, of the CME. The half width, $\lambda$, is the angle between one boundary and $\phi_{\text {Earth }}$. The blue arrow indicates the direction to Earth.

images are selected in such a way that the CME front is clearly visible in the coronagraph images of all the viewpoints. Furthermore, we try to fit the CME at times when the front is already far out in both STA and STB COR2 images. Table 2 additionally contains the GCS parameters (Lon, Lat, TA, AR, HA). Also the half width, $\lambda$, and the CME ecliptic propagation angle, $\phi$, relative to Earth $\left(\phi_{\text {Earth }}\right)$, and relative to the two STEREO spacecraft ( $\phi_{\mathrm{STA}}$ and $\phi_{\mathrm{STB}}$ ) obtained from EAGEL are given. Lon is the longitude (here given in Stonyhurst coordinates) and Lat the latitude of the apex of the idealized hollow croissant shaped model. The tilt angle (TA) defines the tilt of the croissant, calculated with respect to the solar equator. The half angle $(H A)$ represents the angle between the center of the foot points and the aspect ratio $(A R)$ is the ratio of the CME size in two orthogonal directions.

Table 2

GCS Parameter Obtained From Fitting the Hollow Croissant Shape to the STEREO and SoHO Coronagraph Images and EAGEL Results

\begin{tabular}{|c|c|c|c|c|c|c|c|c|c|c|}
\hline \multirow[b]{2}{*}{ No. } & \multirow[b]{2}{*}{ Date } & \multicolumn{5}{|c|}{ GCS parameter } & \multicolumn{4}{|c|}{ EAGEL results } \\
\hline & & Lon $\left(^{\circ}\right)$ & Lat $\left({ }^{\circ}\right)$ & $\mathrm{TA}\left({ }^{\circ}\right)$ & $\operatorname{AR}\left({ }^{\circ}\right)$ & $\mathrm{HA}\left({ }^{\circ}\right)$ & $\lambda\left({ }^{\circ}\right)$ & $\phi_{\text {Earth }}\left({ }^{\circ}\right)$ & $\phi_{\text {STA }}\left({ }^{\circ}\right)$ & $\phi_{\mathrm{STB}}\left({ }^{\circ}\right)$ \\
\hline 1 & February 03, 2010, 15:54 & 355 & -17 & -1 & 0.33 & 30 & 36 & -4 & 67 & 68 \\
\hline 2 & March 19, 2010 17:39 & 23 & -12 & -7 & 0.29 & 19 & 30 & 22 & 44 & 93 \\
\hline 3 & April 03, 2010, 12:39 & 7 & -19 & 15 & 0.39 & 30 & 38 & 9 & 58 & 81 \\
\hline 4 & April 08, 2010 06:39 & 1 & -10 & -20 & 0.28 & 30 & 31 & -2 & 70 & 69 \\
\hline 5 & May 23, 2010 20:39 & 6 & 2 & -15 & 0.48 & 18 & 35 & -6 & 65 & 76 \\
\hline 6 & October 26, 2010 14:39 & 18 & -35 & -28 & 0.51 & 30 & 18 & -11 & 95 & 69 \\
\hline 7 & January 30, 2011 21:24 & 351 & -18 & -20 & 0.33 & 12 & 24 & -11 & 97 & 82 \\
\hline 8 & February 15, 2011 04:08 & 10 & -10 & 27 & 0.87 & 29 & 49 & 10 & 77 & 104 \\
\hline 9 & September 06, 2011 23:39 & 29 & 20 & -90 & 0.49 & 30 & 26 & 29 & 74 & 124 \\
\hline 10 & January 23, 2012 04:39 & 19 & 41 & 64 & 0.77 & 55 & 37 & 9 & 99 & 123 \\
\hline 11 & June 14, 2012 14:54 & 360 & -28 & 11 & 0.90 & 30 & 53 & 1 & 116 & 117 \\
\hline 12 & July 12, 2012 17:54 & 8 & -12 & 68 & 0.46 & 30 & 26 & 14 & 106 & 129 \\
\hline
\end{tabular}

Note. Date: time set in EAGEL to perform the reconstruction. Lon: longitude (Stonyhurst coordinates), Lat: latitude, $T A$ : tilt angle, $A R$ : aspect ratio, $H A$ : half angle from GCS. The remaining values are based on the ecliptic cut from EAGEL: $\lambda$ : half width of the CME, $\phi_{\text {Earth }}, \phi_{\text {STA }}, \phi_{\text {STB }}$ : propagation direction with respect to Earth, STA, STB, respectively. 
When comparing Lon (longitude from GCS reconstruction) and $\phi_{\text {Earth }}$ (longitude relative to Earth from the ecliptic cut), it can be seen that the propagation direction obtained from the ecliptic cut is quite comparable to (within $5^{\circ}$ of) the propagation direction from the GCS reconstruction. Only for events \#6 and \#10 we find differences of about $30^{\circ}$ and $10^{\circ}$, respectively. The reason can be found in the combination of low/high latitude and large tilt angle. Therefore, the part within the ecliptic plane does not correspond well to the main propagation direction resulting from GCS reconstruction for these two CMEs.

\subsection{WSA/HUX Model}

In the following paragraph, we summarize the main characteristics of the numerical framework used here for modeling the physical conditions in the evolving ambient solar wind flow. For this study, we make use of the framework shown in Reiss et al. $(2019,2020)$, but the components of this framework were developed by Wang and Sheeley (1995), Arge et al. (2003), Riley and Lionello (2011), and Owens and Riley (2017). Specifically, we use magnetic maps of the photospheric field from Global Oscillation Network Group (GONG) provided by the National Solar Observatory (NSO) as input to magnetic models of the solar corona. Using the Potential Field Source Surface model (PFSS; Altschuler \& Newkirk, 1969; Schatten et al., 1969) and the Schatten current sheet model (SCS; Schatten, 1971) we compute the global coronal magnetic field topology. While the PFSS model attempts to find the potential magnetic field solution in the corona with an outer boundary condition that the field is radial at the source surface at $2.5 R_{\odot}$, the SCS model in the region between 2.5 and $5 R_{\odot}$ accounts for the latitudinal invariance of the radial magnetic field as observed by Ulysses (Wang \& Sheeley, 1995). From the global magnetic field topology, we calculate the solar wind conditions near the Sun using the established Wang-Sheeley-Arge (WSA) model. To map the solar wind solutions from near the Sun to Earth, we use the Heliospheric Upwind eXtrapolation model (HUX). This model simplifies the fluid momentum equation as much as possible, by neglecting the pressure gradient and the gravitation term in the fluid momentum equations as proposed by Riley and Lionello (2011). The HUX model solutions match the dynamical evolution explored by global heliospheric MHD codes fairly well while having low processor requirements. An example of the ambient solar wind, modeled by WSA/HUX combination, is shown in Figure 2.

\subsection{ELEvoHI Ensemble Modeling}

ELEvoHI uses HI time-elongation profiles of CME fronts and assumes an elliptical shape for those fronts to derive their interplanetary kinematics (detailed information about the underlying Ellipse Conversion method can be found in Rollett et al. (2016). The tracking of each CME was done manually using ecliptic time-elongation maps (j-maps; Davies et al., 2009; Sheeley et al., 1999), generated by extracting ecliptic data from STA and STB HI images. Transients, like CMEs, appear as a bright feature in the j-maps. To extract the time-elongation profiles, we use the SATPLOT tool implemented in IDL ${ }^{\mathrm{TM}}$ SolarSoft. It allows any user to measure the elongation, which is defined as the angle between the Sun-observer (STA or STB) line and the CME front. ELEvoHI converts the resulting time-elongation profiles to time-distance profiles, assuming an elliptic frontal shape using the ELEvoHI built-in procedure ELlipse Conversion (ELCon; Rollett et al., 2016).

ELEvoHI accounts for the effect of the drag force exerted by the ambient solar wind, which is incorporated in the model. The drag force is an essential factor influencing the dynamic evolution of CMEs in the heliosphere. Within ELEvoHI, the time-distance track is fitted using a drag-based equation based on the drag-based model (DBM) given in Vršnak et al. (2013). The user has to define the start-point and end-point for the DBM fit (usually around $30-100 R_{\odot}$ ) in the time-distance profile. In order to account for the de-/ acceleration of the CME due to drag, an estimate of the ambient solar wind speed is needed. Here, we make use of the WSA/HUX model (see Section 3.2), which provides the ambient solar wind conditions for a full Carrington rotation (see Figure 2). We only consider the part of the full map according to the start-point and end-point selected by the user, and the CME propagation direction and half width from EAGEL. From this area, surrounded by the white box in Figure 2, we take the median of the solar wind speed and define the uncertainties to be $\pm 100 \mathrm{~km} \mathrm{~s}^{-1}$, based on a study by Reiss et al. (2020). They considered 9 years (mid-2006 to mid-2015) and report a mean absolute error of the WSA solar wind speed prediction with respect to the in situ speed of $91 \mathrm{~km} \mathrm{~s}^{-1}$. The obtained ambient solar wind speed with its uncertainty is split into steps of 


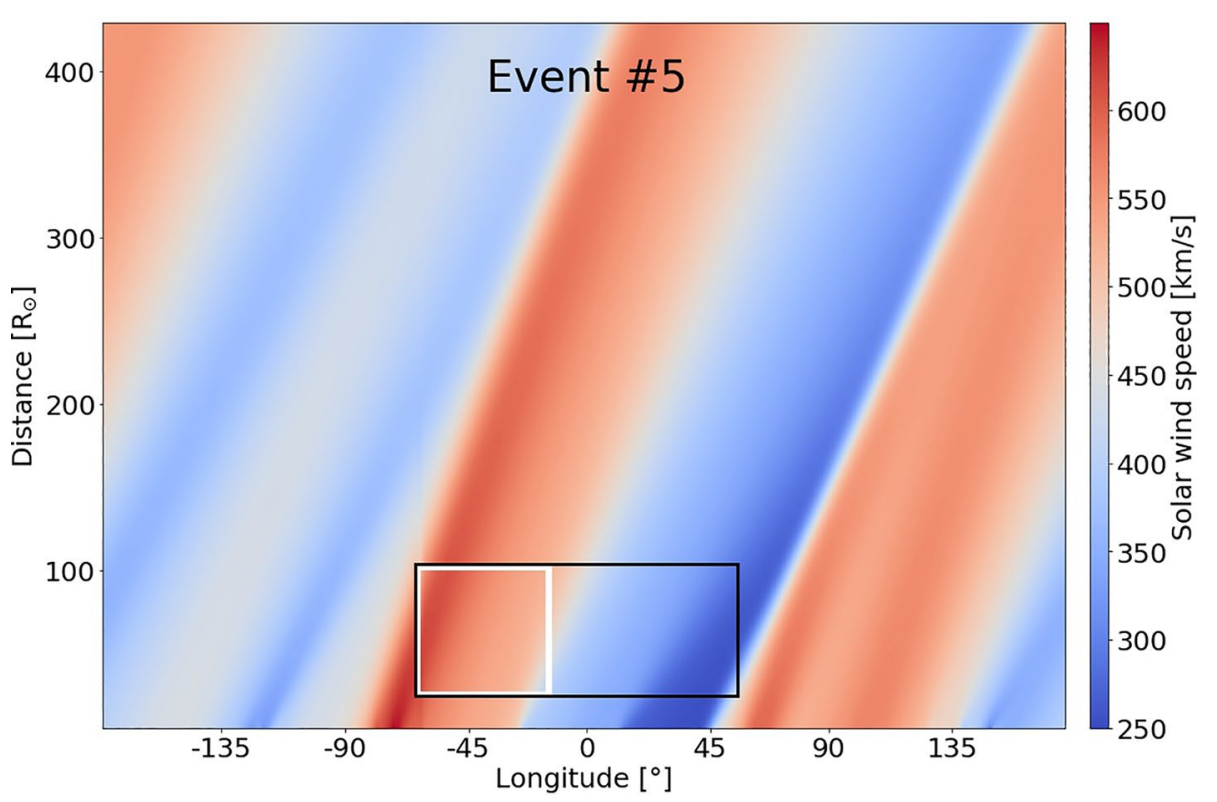

Figure 2. Ambient solar wind speed provided by the WSA/HUX model for event \#5. The white box defines the area that is used to calculate an estimate of the ambient solar wind speed for the ensemble member of ELEvoHI corresponding to the minimum propagation direction $\left(\phi_{\mathrm{STA}}=56^{\circ}\right)$ with the maximum half width $\left(\lambda=50^{\circ}\right)$. The black box indicates the total area based on all the ensemble members of ELEvoHI for this event. The longitude of $0^{\circ}$ corresponds to the longitude of Earth. WSA/HUX, Wang-Sheeley-Arge/Heliospheric Upwind eXtrapolation; ELEvoHI, ELlipse Evolution model based on Heliospheric Imager observations.

$25 \mathrm{~km} \mathrm{~s}^{-1}$, which gives nine different input speeds to ELEvoHI. For each of the nine input speeds, DBM fitting is performed. ELEvoHI then selects the combination of drag parameter and ambient solar wind speed that best fits the time-distance profile for each ensemble member (for a detailed description see Rollett et al., 2016). The selected drag parameter and solar wind speed are assumed to be valid for the full CME front during the propagation in the heliosphere.

Since ELEvoHI is a 2D model, we are only interested in the propagation of a CME in the ecliptic plane. $\phi$ and $\lambda$, in this plane, are provided by EAGEL (see Section 3.1). The inverse ellipse aspect ratio, $f$, defines the shape of the assumed CME front in the ecliptic plane, where $f=1$ represents a circular front, while $f<1$ corresponds to an elliptical CME front (with the semimajor axis perpendicular to the propagation direction).

To run ELEvoHI in ensemble mode, we vary $\phi, \lambda$, and $f$. A details description can be found in Amerstorfer et al. (2018) and the code is available online (see Section 6). $\phi$ and $\lambda$ vary over a range of $\pm 10^{\circ}$ from their values obtained from EAGEL, with a step size of $2^{\circ}$ and $5^{\circ}$, respectively. This range is defined based on a study by Mierla et al. (2010), who report an uncertainty in the parameters when different users manually perform GCS reconstruction. Note that the propagation direction and the half width obtained from EAGEL are rounded to even numbers and to whole tens, respectively. For $f$ we set a fixed range from 0.7 to 1.0 ( 0.1 step size). Thus, we get a total of 220 ensemble members for one ELEvoHI event (i.e., 11 values of $\phi$, 5 values of $\lambda$, and 4 values of $f$ ). For each ensemble member we select a different sector from the ambient solar wind provided by the WSA/HUX model combination according to the propagation direction, half width, start-point, and end-point. In Figure 2, the WSA/HUX model results for event \#5 are shown. The white box indicates the area from which the ambient solar wind speed for one individual run of ELEvoHI is computed. Shown is the area for the minimum propagation direction, $\phi_{\mathrm{STA}}$ of $56^{\circ}$ with a $\lambda$ of $50^{\circ}$. For each ensemble member, the area surrounded by the white box is slightly different according to $\phi$ and $\lambda$. The black box plotted indicates the total area based on all ELEvoHI ensemble members for this event.

Running ELEvoHI in ensemble mode enables us to calculate a mean and a median predicted CME arrival time and also to define an uncertainty. In addition, we can give a probability for whether a CME is likely to hit Earth or not. When all of the 220 ensemble members predict an arrival at Earth, we assume the predicted likelihood of an Earth hit to be $100 \%$. 
Table 3

List of Predicted Median Arrival Times (Date) and the Standard Deviation (SD) Based on STA and STB Observations, Respectively

\begin{tabular}{|c|c|c|c|c|c|c|c|c|}
\hline No. & Date STA & $\mathrm{SD}_{\text {STA }}(\mathrm{h})$ & Date STB & $\mathrm{SD}_{\text {STB }}(\mathrm{h})$ & STA - STB (h) & $v_{\text {STA }}\left(\mathrm{km} \mathrm{s}^{-1}\right)$ & $v_{\text {STB }}\left(\mathrm{km} \mathrm{s}^{-1}\right)$ & $v_{\text {STA-STB }}\left(\mathrm{km} \mathrm{s}^{-1}\right)$ \\
\hline 1 & February 07, 2010 11:24 & 1.5 & February 07, 2010 20:24 & 2.1 & -9.0 & $455 \pm 17$ & $395 \pm 11$ & 60 \\
\hline 2 & March 24, 2010 07:17 & 9.1 & March 24, 2010 16:40 & 4.1 & -9.5 & $401 \pm 32$ & $351 \pm 11$ & 50 \\
\hline 3 & April 05, 2010 13:23 & 2.5 & April 05, 2010 16:06 & 0.4 & -2.7 & $649 \pm 37$ & $625 \pm 5$ & 24 \\
\hline 4 & April 11, 2010 16:07 & 0.6 & April 12, 2010 00:12 & 5.1 & -8.1 & $443 \pm 6$ & $391 \pm 33$ & 52 \\
\hline 5 & May 27, 2010 17:36 & 1.9 & May 28, 2010 02:26 & 1.2 & -8.8 & $455 \pm 9$ & $407 \pm 9$ & 48 \\
\hline 6 & October 30, 2010 11:24 & 1.4 & October 30, 2010 04:43 & 7.1 & 6.7 & $432 \pm 7$ & $476 \pm 45$ & -44 \\
\hline 7 & February 04, 2011 01:08 & 2.4 & February 03, 2011 22:24 & 7.3 & 4.5 & $387 \pm 9$ & $446 \pm 34$ & -59 \\
\hline 8 & February 18, 2011 06:22 & 2.8 & February 18, 2011 10:34 & 6.1 & -4.3 & $478 \pm 18$ & $407 \pm 50$ & 71 \\
\hline 9 & September 10, 2011 18:55 & 14.9 & September 10, 2011 09:48 & 5.4 & 9.1 & $396 \pm 46$ & $430 \pm 18$ & -34 \\
\hline 10 & January 24, 2012 17:49 & 4.0 & January 24, 2012 13:29 & 3.6 & 4.3 & $793 \pm 103$ & $982 \pm 150$ & -189 \\
\hline 11 & June 16, 2012 15:47 & 3.8 & June 16, 2012 07:53 & 5.2 & 7.9 & $712 \pm 72$ & $749 \pm 143$ & -37 \\
\hline 12 & July 14, 2012 22:16 & 4.9 & July 14, 2012 18:53 & 3.7 & 3.5 & $658 \pm 80$ & $579 \pm 28$ & 89 \\
\hline
\end{tabular}

Note. STA - STB gives the difference between the predicted median arrival times. $v$ is the predicted median arrival speed with the standard deviation and $v_{\text {STA-STB }}$ is the difference in arrival speed between STA and STB predictions.

\section{Results}

We perform ELEvoHI ensemble modeling for 12 CMEs between February 2010 to July 2012 (see Table 1) and compare the predicted arrival times based on STA and STB HI observations with each other. The CMEs propagated close to the ecliptic plane and showed clear in situ signatures at L1. A prerequisite for the chosen CMEs was that the CMEs could be tracked unambiguously in both STA and STB HI j-maps.

In Table 3, we list the predicted ensemble median arrival times and speeds with their standard deviation for each CME under study. It further contains the difference between the predictions from the two vantage points. We find that the predicted arrival times for STA and STB can deviate by up to $9.5 \mathrm{~h}$ while the mean difference is $6.5 \mathrm{~h}$. The mean difference in the arrival speed is $63 \mathrm{~km} \mathrm{~s}^{-1}$, with an exceptionally large discrepancy of $189 \mathrm{~km} \mathrm{~s}^{-1}$ for event \#10.

The largest arrival time differences are found for events \#2 and \#9. The arrival probability, based on the number of ensemble members that are predicted to hit Earth, is $79 \%$ for event \#2 and only $56 \%$ for event \#9. According to their relatively large angle of propagation with respect to the Sun-Earth line, the CMEs $\# 2\left(\phi_{\text {Earth }}=22^{\circ}, H A=30^{\circ}\right)$ and $\# 9\left(\phi_{\text {Earth }}=30^{\circ}, H A=30^{\circ}\right)$ are considered as "flank hits." In such cases, ELEvoHI tends to predict the CME arrival time to be later than detected in situ. The reason may be found in the assumed circular CME front for $f=1.0$. For future versions of ELEvoHI, we will consider different approaches to tackle such extreme delays for flank encounters e.g., by changing the values for $f$ (from 0.4 to 0.7). Braga et al. (2020) found a value of $f \sim 0.6$ for most of the CMEs in their study.

Event \#11 occurred on June 14, 2012 and was studied e.g., by Kubicka et al. (2016) who report two preceding CMEs. However, the WSA/HUX model does not provide the ambient solar wind conditions with preceding CMEs included and is therefore most probably not suitable for interaction events. The events \#1, \#4, and \#5 also show large differences in the predicted arrival times based on STA and STB observations. However, these differences are most certainly related to large variance in the modeled ambient solar wind speeds that are used as input to ELEvoHI (see Section 4.2 and Figures 5 and 6). 


\subsection{Tracking Different Parts of the CME Front}

It is important to keep in mind that different parts of the CME front are tracked in STA and STB HI images. This leads to different input conditions to the ELEvo propagation model for STA and STB. ELEvoHI is designed to take HI tracks for the same CME from different viewpoints. Ideally, predictions should give the same CME speed and direction in both cases. One problem is, however, that the CME is not behaving as a single coherent entity, but is instead moving with different speeds at different longitudes (Owens et al., 2017), which is not incorporated within ELCon nor in any other HI conversion method (e.g., SSE, FP, and $\mathrm{HM})$.

Figure 3 presents two snapshots of a movie for event \#4, with the ambient solar wind provided by WSA/ HUX model combination and the positions of various spacecraft and planets. The elliptical CME fronts from one ensemble member based on STA and STB observations are shown in red and blue, respectively. The gray lines from the two STEREO spacecraft to the elliptical CME fronts are plotted. These tangents correspond to the elongations of the leading edge of the CME at these times. At the end of these lines, we add a point, which is the "tangent point" at each time step. Over the course of the simulation, these points trace out curved lines, in red and blue for STA and STB, respectively. From Figure 3, it is obvious that, in the near-Sun part of the HI FOV, the observed leading edge is close to the apex of the idealized CME front for both STEREO spacecraft. As the CME propagates, the tangent point, i.e., the part of the CME with the greatest elongation seen by STA and STB progressively moves out to the flanks of the ellipse. Based on the observations of these tangent points, the prediction for the whole front is conducted. Hence, the apex of the CME is, if at all, only observed for a short period of time. In order to get an estimate of the CME Earth arrival we have to assume a designated shape of the CME front, which is in our case, an ellipse. As shown by Owens et al. (2017) this assumption might not be valid since the CME interacts with the ambient solar wind.

\subsection{Effect of the Ambient Solar Wind}

When considering different points along the idealized elliptical CME front, it is noteworthy that the ambient solar wind speeds at these points would likely be different. Furthermore, the part of the CME front corresponding to the greatest elongation as seen by STA and STB (i.e., the points corresponding to the tangent to the CME front) would propagate in different ambient solar wind conditions. In Figure 4, the modeled time-elongation profiles of the tangent points seen from STA (top panel) and STB (bottom panel) for event \#4 are shown.

These profiles are obtained from one modeled ensemble member of the ELEvoHI prediction, separately for STA and STB (see Figure 3), and are therefore available from April 08, 2010 11:00 until April 14 2010, 10:00. As long as the CME front could be tracked in HI images (until about April 10, 2010 01:00), the plotted profiles are consistent with the measured HI time-elongation profiles, obtained using the SATPLOT tool. The colors represent the speed of the ambient solar wind at the corresponding points. Due to the propagation of the modeled CME in the heliosphere, the elongation of the tangent point ranges from roughly $8^{\circ}$ to about $92^{\circ}$ and the speed of the ambient solar wind at these points ranges from 330 to $500 \mathrm{~km} \mathrm{~s}^{-1}$, with a maximum speed of $530 \mathrm{~km} \mathrm{~s}^{-1}$ at about $66^{\circ}$, for STA (top panel in Figure 4). The range of the elongation is similar for $\operatorname{STB}\left(6^{\circ}-91^{\circ}\right)$ but the ambient solar wind speed ranges only from $\approx 450$ to $\approx 365 \mathrm{~km} \mathrm{~s}^{-1}$.

In the previous paragraph, we considered the ambient solar wind speed at the tangent point for one ensemble member. Additionally, we examine the distribution of the ambient solar wind speed considered for all ensemble members (see black boxes in Figures 2 and 5) that are used as input to ELEvoHI for a single CME. From the areas framed by the black boxes, we calculate the standard deviation and correlate those to the absolute values of the difference between STA and STB arrival time predictions for each event (see Figure 6). This gives us the possibility to check the influence of the ambient solar wind on the arrival time differences. We obtain a Pearson correlation coefficient of $c c=0.52$ for all events under study. However, when excluding events \#2 and \#9, which are considered as "flank hits," and excluding event \#11 (CMECME interaction event), the Pearson correlation coefficient increases to $c c=0.92$. This indicates that a more structured ambient solar wind (i.e., a larger standard deviation) leads to a larger difference between STA and STB arrival time prediction. 
(a)

\section{Event \#4 20100408 13:00:00}
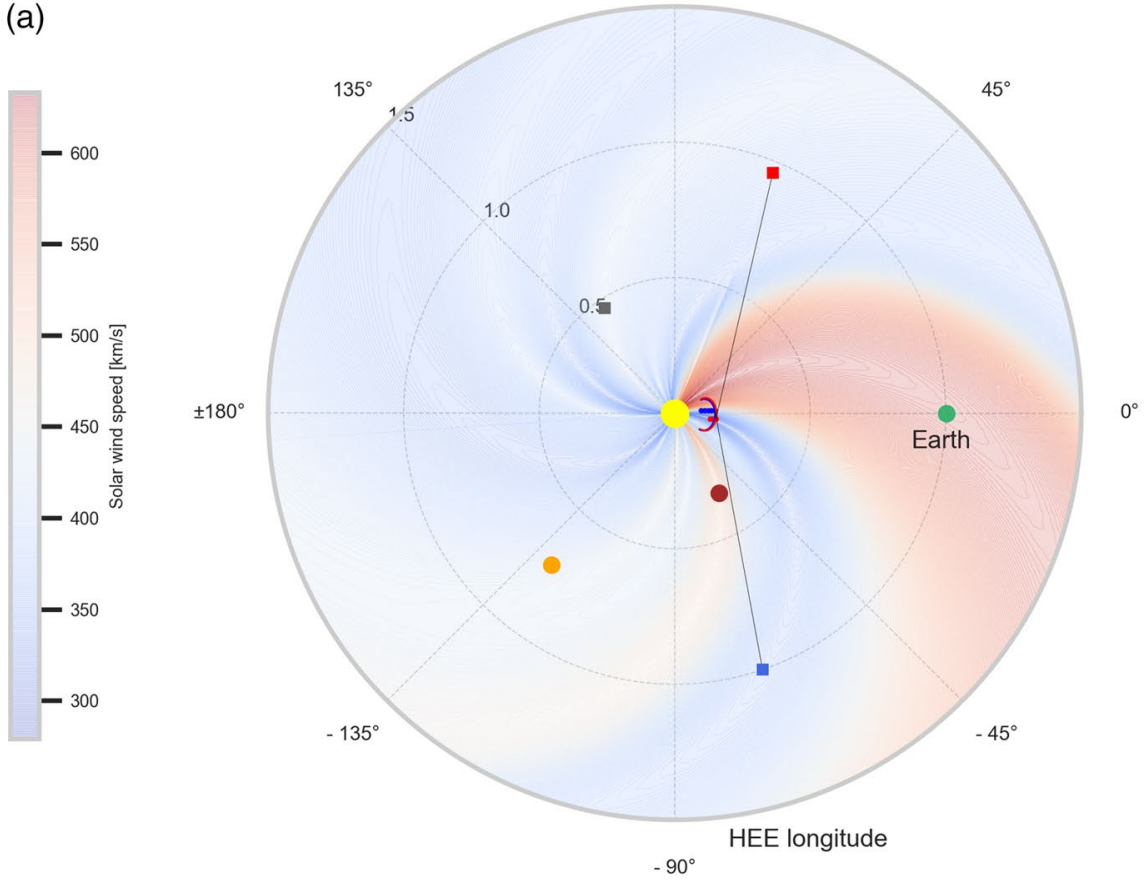

Mercury MESSENGER

Venus STEREO-A STEREO-B Earth

(b)

Event \#4 $20100411 \quad 21: 00: 00$
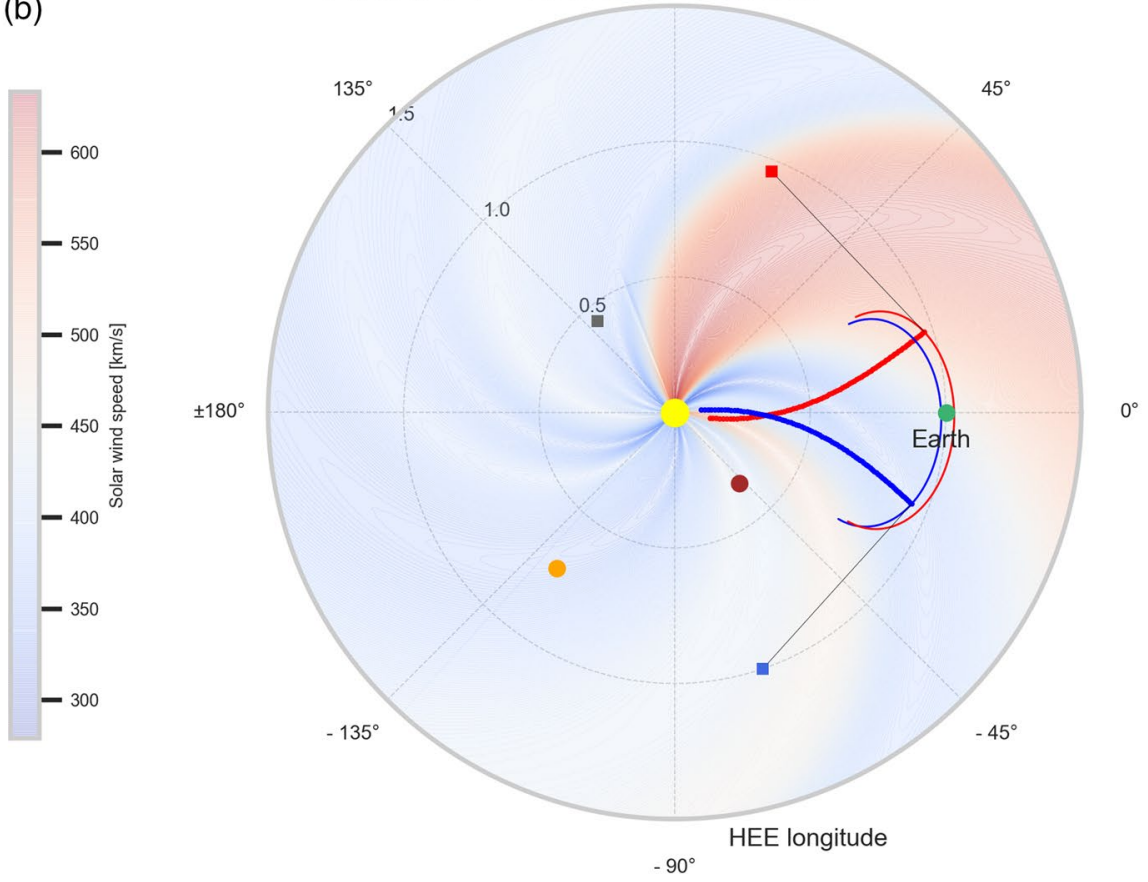

Mercury

MESSENGER

Venus

STEREO-A

STEREO-B

Earth 


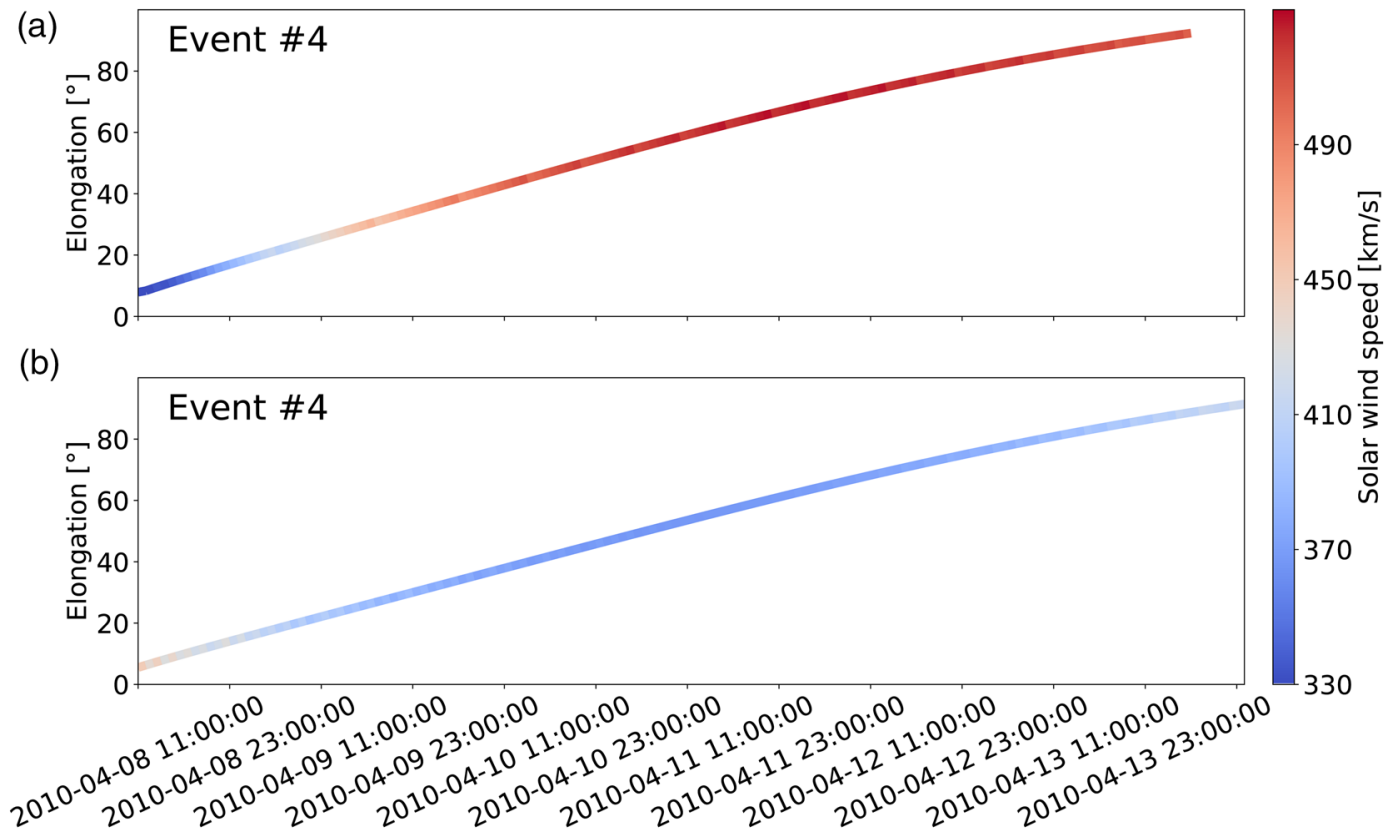

Figure 4. Ambient solar wind speed at the tangent points for event \#4. Plotted are time series of the elongation angles of the tangent points as seen from STA (top panel) and STB (bottom panel) color-coded according to the speed of the ambient solar wind at that tangent point. STA, STEREO Ahead; STB, STEREO Behind.

\subsection{Comparison to In Situ Arrivals}

Figure 7 shows the distributions of the arrival time and arrival speed differences with respect to the in situ arrivals for all ensemble members for each CME. Blue and orange correspond to STB and STA ensemble predictions, respectively. The black horizontal lines indicate the median values of each distribution. When comparing the median predicted arrival times to the in situ arrivals, we obtain a mean absolute error (MAE) over all events of $7.5 \pm 9.5 \mathrm{~h}$ and a root mean square error (RMSE) of $\approx 10.4 \mathrm{~h}$. A mean error $(\mathrm{ME})$ of $\approx 4 \mathrm{~h}$ indicates, in this setup, that ELEvoHI tends to predict the arrivals too late. The highest arrival time discrepancy is found for event \#9 where the prediction based on STA is $31 \mathrm{~h}$ too late. When comparing the median predicted arrival speeds to the in situ speeds we get an MAE of $87 \pm 111 \mathrm{~km} \mathrm{~s}^{-1}$, an RMSE of $\approx 123 \mathrm{~km} \mathrm{~s}^{-1}$ and an ME of $\approx 52 \mathrm{~km} \mathrm{~s}^{-1}$. The highest speed difference is found for the STB prediction of event \#10, overestimating the arrival speed by $369 \mathrm{~km} \mathrm{~s}^{-1}$.

Interestingly, event \#10 gives an accurate predicted arrival time, even though the predicted arrival speed is highly overestimated. When performing GCS reconstruction, we obtain a high latitude and a large tilt angle for this CME meaning that the 3D propagation direction differs from that in the ecliptic plane (see Table 2). As already mentioned, event \#11 is a CME-CME interaction event which explains the large discrepancy especially for the predicted arrival speed. The reason might be found in an extremely low drag due to preconditioning in the interplanetary space (Liu et al., 2014; Rollett et al., 2014; Temmer \& Nitta, 2015).

\section{Discussion and Conclusions}

We present the ELEvoHI ensemble modeling results for 12 CMEs, occurring between February 2010 and July 2012, that were observed by both STEREO spacecraft. This study mainly focuses on the difference of the modeled arrival time and arrival speed when using STA and STB HI observations, separately. We find

Figure 3. Two snapshots of the CME propagation for one ensemble member based on STA (red) and STB (blue) observations for event \#4. The ambient solar wind is computed using the WSA/HUX model combination. The elliptical CME fronts from one ensemble member based on STA and STB observations are shown in red and blue, respectively. The thick curved lines in red and blue show the intercept of the idealized elliptical front of the CME and the tangent (gray lines) for each time step over the course of the simulation for STA and STB, respectively. Link to the movie (https://figshare.com/articles/media/20110130_AB_ tangent_movie/13077608). CME, coronal mass ejection; STA, STEREO Ahead; STB, STEREO Behind. 

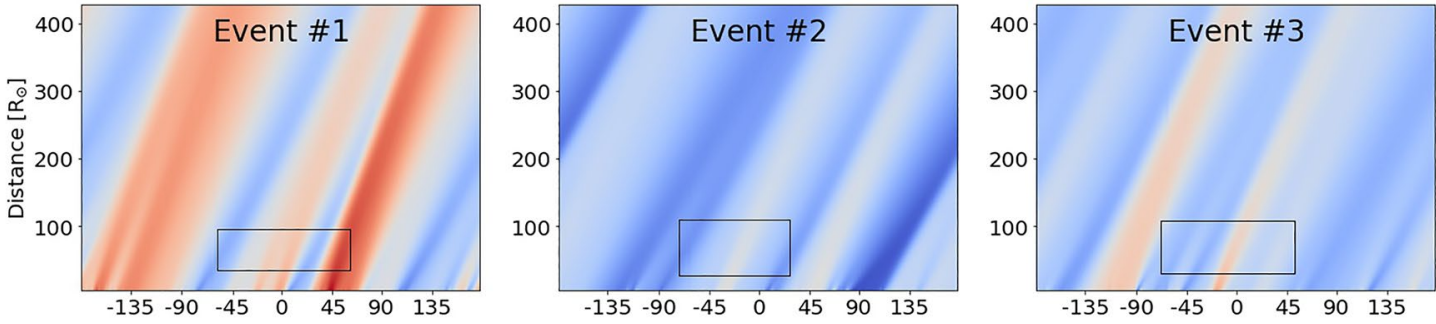

650
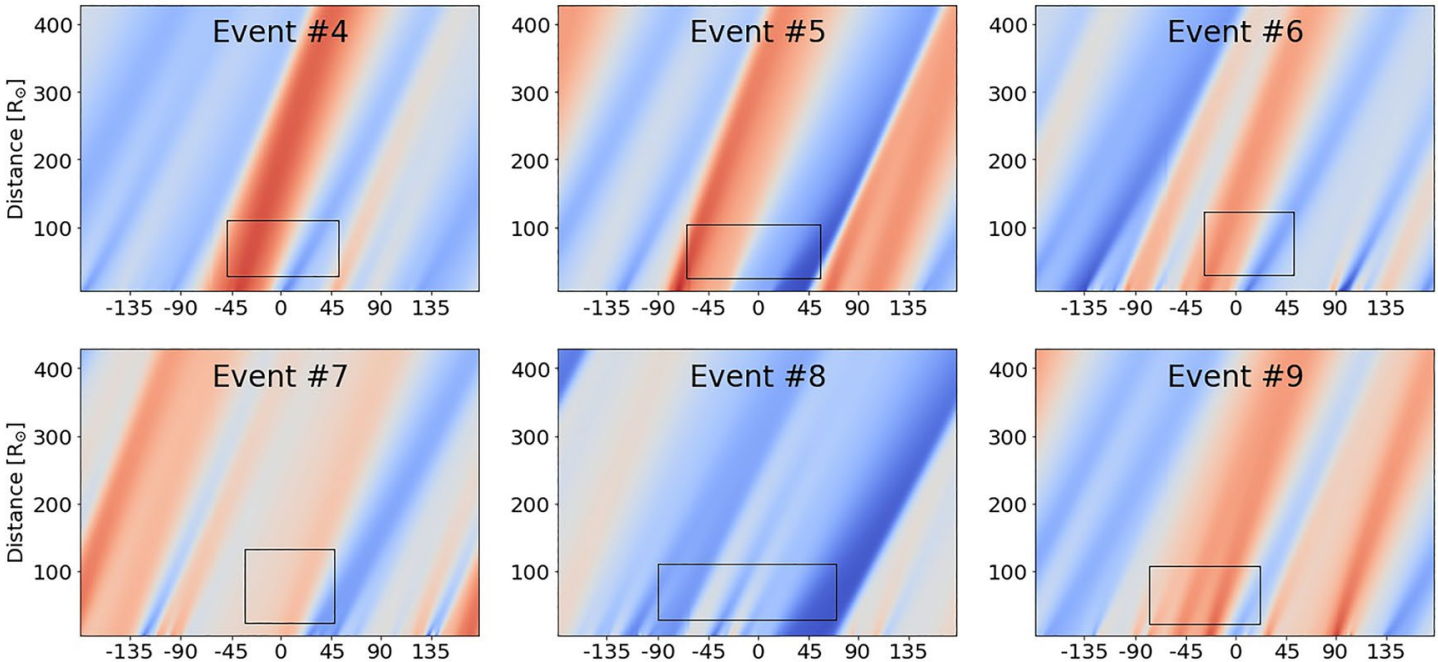

$-600$
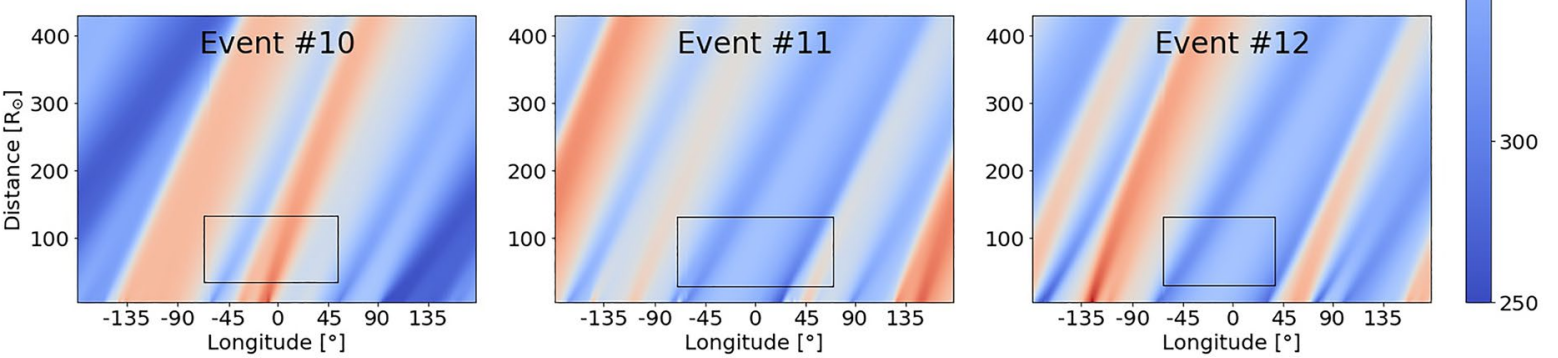

Figure 5. Ambient solar wind speed provided by the WSA/HUX model combination for all 12 events under study. The black boxes define the areas that are used to estimate how structured the ambient solar wind is for each CME. Longitude of $0^{\circ}$ corresponds to the longitude of Earth. CME, coronal mass ejection.

on average a difference of $6.5 \mathrm{~h}$ between arrival time predictions from the two spacecraft but the largest difference is about $9.5 \mathrm{~h}$ for event \#9. For the arrival speed we find a mean difference between STA and STB predictions of $63 \mathrm{~km} \mathrm{~s}^{-1}$ with a maximum difference of $189 \mathrm{~km} \mathrm{~s}^{-1}$ for event \#10.

ELEvoHI tends to predict the arrival time later than observed for CMEs that are considered as "flank hits" (event \#2 and event \#9). For such events the propagation direction with respect to Earth is larger than $20^{\circ}$, and not all of the ensemble members predict an Earth impact. The reason for the late arrival prediction may be found in the assumed circular shape (for $f=1.0$ ) and the highly curved flanks.

We provide two CME arrival time and arrival speed predictions, from STA and STB observation, for the same CME to examine the reasons for the discrepancy between these two predictions. We find, that the CME front propagates in different ambient solar wind conditions when observed in STA and STB HI images. However, the kinematics of the CME front obtained e.g., by STA data are used for modeling of the whole CME front, including the Earth-directed part. The same applies for predictions based on STB data, which is the reason for the differences in the predicted arrival times based on STA and STB observations.

We further see, that an ambient solar wind exhibiting a high variance within the area used for ELEvoHI model predictions leads to larger discrepancies between STA and STB model predictions. We obtain a Pear- 


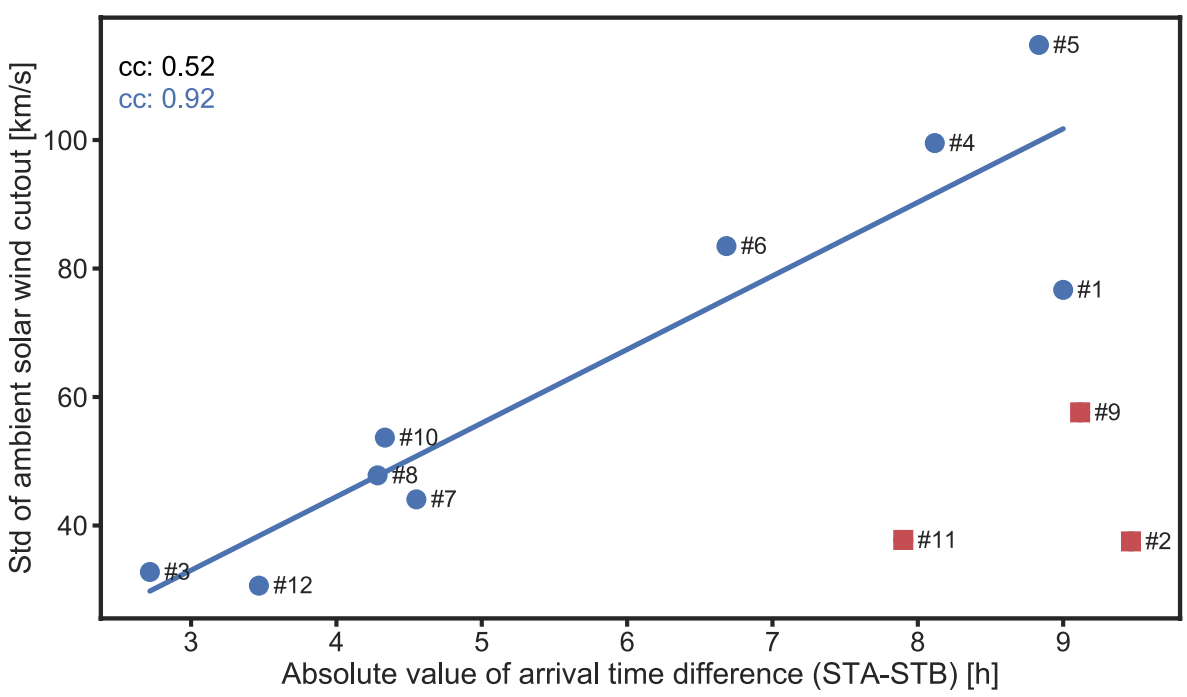

Figure 6. Standard deviation of the ambient solar wind versus the arrival time difference between STA and STB predictions. The Pearson correlation coefficient for all events under study (black) is calculated. In blue, we present the Pearson correlation coefficient and a linear fit when excluding the outliers (indicated by the red boxes), that is, flank hits (events \#2 and \#9) and the CME-CME interaction event (event \#11). STA, STEREO Ahead; STB, STEREO Behind; CME, coronal mass ejection.

son correlation coefficient $(c c=0.92)$, when excluding flank hits (events \#2 and event \#9) and the CMECME interaction event (event \#11). Furthermore, we assume that in such cases the CME front is more likely to deform from an idealized elliptical shape due to interaction with the ambient solar wind (Owens et al., 2017; Riley \& Crooker, 2004).

The current CME forecasting abilities in the community are summarized in Riley et al. (2018). They analyzed CME forecasts that have been submitted to the Community Coordinated Modeling Center (CCMC) scoreboard from 2013 to mid-2018. The CCMC scoreboard is a platform provided to scientists to compare their forecasts with each other in real-time. Riley et al. (2018) found that the CME shock arrival times for all models combined are predicted on average within $\pm 10 \mathrm{~h}$ but with standard deviations of sometimes $>20 \mathrm{~h}$. The best model performance was found for the WSA-ENLIL + Cone model (Odstrcil et al., 2004), run by the UK Met Office, having a bias of $1 \mathrm{~h}$, an MAE of $13 \mathrm{~h}$, and a standard deviation of $15 \mathrm{~h}$. The results of this study are similar to the findings of Riley et al. (2018) when comparing the modeled arrival times to the actual arrivals of CMEs, as determined from in situ measurements. Here, we only perform hindcasts of CME arrivals. For the 24 arrival predictions (12 based on STA and 12 based on STB observations), we obtain an MAE of $7.5 \pm 9.5 \mathrm{~h}$, an RMSE of $\approx 10.4 \mathrm{~h}$, and an ME of $\approx 4 \mathrm{~h}$ for the arrival time. For the arrival speed, we get an MAE of $87 \pm 111 \mathrm{~km} \mathrm{~s}^{-1}$, an RMSE of $\approx 123 \mathrm{~km} \mathrm{~s}^{-1}$, and an ME of $\approx 52 \mathrm{~km} \mathrm{~s}^{-1}$.

As already mentioned, event \#11 is a CME-CME interaction event studied for example, by Kubicka et al. (2016). This CME was closely preceded by two other CMEs that erupted one and two days before this event and that altered the conditions in the heliosphere. The arrival time prediction for this CME is about $11 \mathrm{~h}$ too early, while the arrival speed is greatly overestimated (by $260 \mathrm{~km} \mathrm{~s}^{-1}$ ) using the ambient solar wind solutions provided by the WSA/HUX model combination. However, this model does not consider preceding CMEs and is likely not valid in such cases. An additional approach to infer the ambient solar wind conditions in the low heliosphere is shown in Barnard et al. (2019). In this study, the authors established a statistical relationship between the solar wind speed in the low heliosphere and the variability in HI images. A recent study by Amerstorfer et al. (2020) focuses on different input parameters to ELEvoHI including three possible methods to infer the ambient solar wind conditions needed by the model. First, the ambient solar wind speed is obtained from in situ measurements at 1 AU. Second, the solar wind speed is based on a statistical approach using 14 years of OMNI data. Third, an estimate of the ambient solar wind speed is 


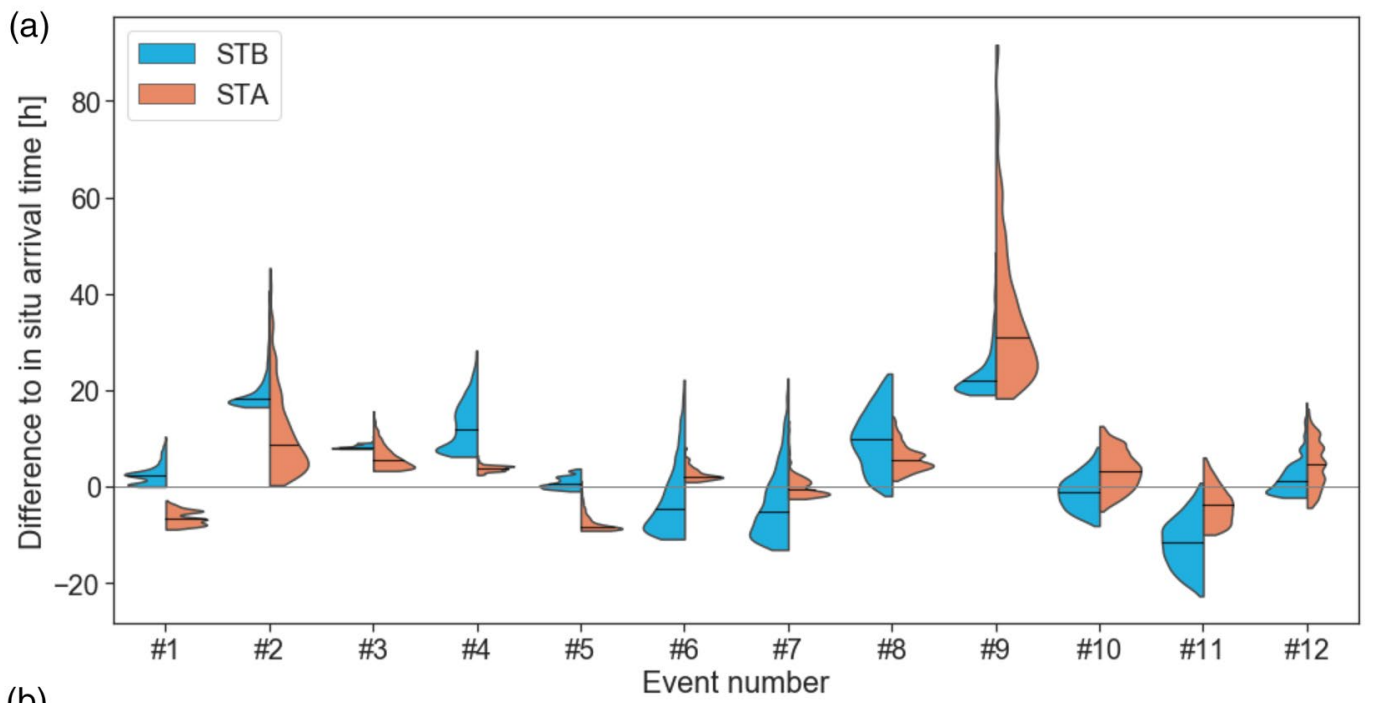

(b)

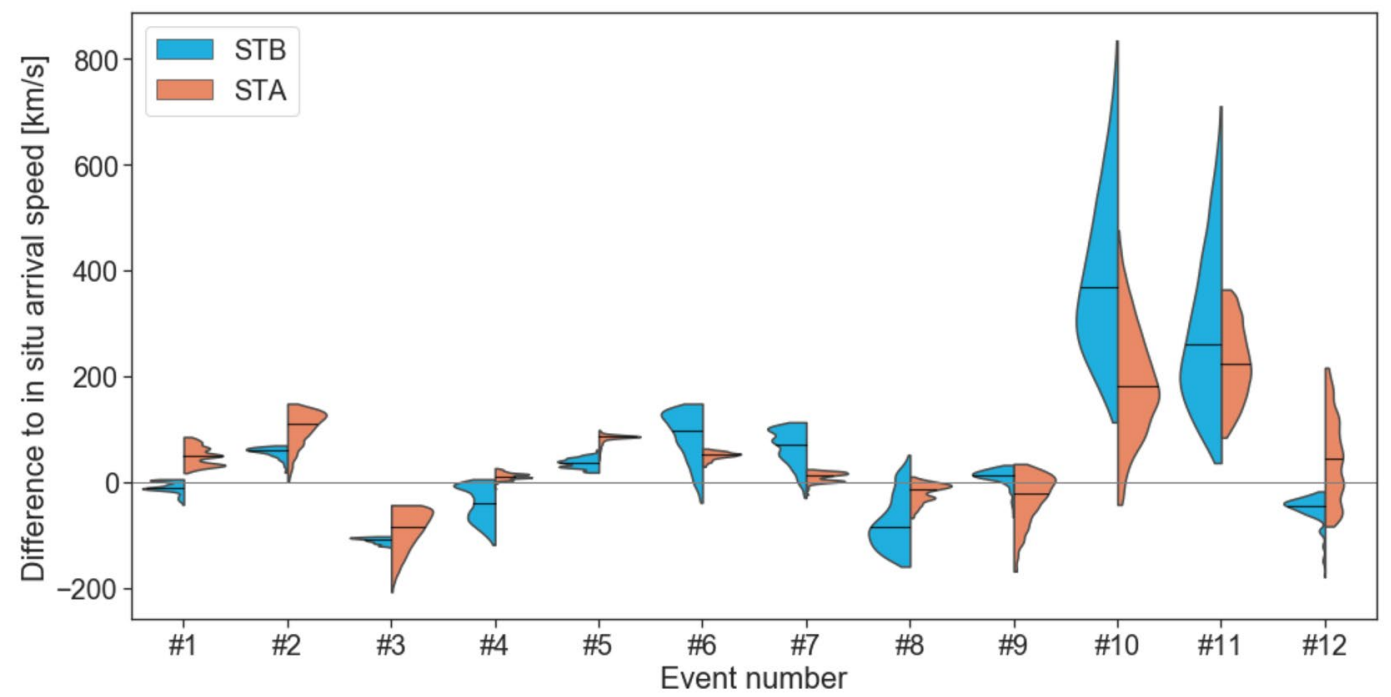

Figure 7. Frequency distributions derived from all ensemble members for the arrival time prediction (top panel) and the arrival speed prediction (bottom panel) based on HI data from STB (blue) and STA (orange), respectively. In the top panel, positive values correspond to a late arrival time prediction while negative values indicate an early arrival prediction. Positive/negative values in the bottom panel indicate an over-/underestimated arrival speed prediction. The black horizontal bars show the median values of the distributions of all the ensemble members for STB and STA. HI, heliospheric imager; STB, STEREO Behind; STA, STEREO Ahead.

obtained from the WSA-HUX model combination. In their study, Amerstorfer et al. (2020) concluded that the third approach provides the best results.

ELEvoHI provides ensemble predictions based on various inputs, namely propagation direction, half width, inverse aspect ratio, and ambient solar wind speed. In the current version, ELEvoHI is not able to react to possible deflections of a CME during its propagation. Furthermore, the elliptical CME shape, once defined by the input parameters, does not change during propagation. This has been shown to be invalid by, for example, Rollett et al. (2014), who performed a case study by combining HI data with in situ data to ascertain the kinematics of the March 7, $2012 \mathrm{CME}$. The authors demonstrated evidence for an asymmetric evolution of the CME, which was caused by the preconditioned ambient solar wind resulting in a different drag regime influencing different parts of the CME. 
CME deflection (e.g., Wang et al., 2004, 2014) and deformation (e.g., Barnard et al., 2017; Kay \& Nieves-Chinchilla, 2020) are important factors when considering CME propagation in the heliosphere. The authors found that the failure to take these factors into account would likely lead to uncertainties in the arrival time and arrival speed prediction. Barnard et al. (2017) additionally showed that different tracks lead to quite different CME arrival time predictions. By using HI observations with better solar wind modeling and varying CME frontal shapes we should be able to improve our current arrival time predictions (Barnard et al., 2020).

A number of studies have taken advantage of stereoscopic HI observations, from the two STEREO spacecraft, to glean information on CME propagation and evolution (e.g., Davies et al., 2013; Liu et al., 2010; Lugaz, 2010; Volpes \& Bothmer, 2015). Braga et al. (2020) studied 14 CMEs using the drag model and a modified version of the ELCon model, to get the CME parameters (e.g., $\phi, \lambda, f$ ) based on HI observations from both STEREO spacecraft simultaneously. For the five events, that are also included in our study, we obtain similar results as Braga et al. (2020). Please note that we run ELEvoHI in ensemble mode in this study. We believe, that a stereoscopic view on CMEs incorporated in ELEvoHI will improve the arrival time predictions substantially. Therefore, we strongly support ESA's L5 mission, equipped with a heliospheric imager (Kraft et al., 2017; Lavraud et al., 2016), and an additional heliospheric imager at L1. Fortunately, the upcoming Earth-orbiting PUNCH mission (launch planned in 2023) will also possess wide-angle whitelight heliospheric imagers, as well as a coronagraph, and will be able to provide additional observations of CMEs. Based on information from these additional vantage points, more accurate CME arrival predictions are likely to be achieved. Since ELEvoHI is ready to be used in near real-time, future HI observations are essential for further CME arrival predictions. STA, currently near L5, will have moved beyond L4 by 2027, so it will be necessary to have heliospheric imagers that are observing the space between Sun and Earth after around 2030.

In a next step, we want to further develop ELEvoHI in such a way that it can combine HI data from two vantage points in order to constrain the $\mathrm{CME}$ and exclude ensemble runs that are not consistent with the observations. Also, the CME shape can be constrained by multiple HI observations and therefore, we aim to make the CME front deformable during the propagation through the heliosphere. Hence, the assumed elliptical CME front would be able to adjust according to the ambient solar wind conditions. It was already shown in previous studies (e.g., Chi et al., 2020; Scott et al., 2019) that ghost fronts in the HI observations can be used to infer the structure of a CME. Using their approach, we also aim to improve our model by verifying and constraining the CME shape.

\section{Data Availability Statement}

\section{Data}

STEREO/HI: https://www.ukssdc.ac.uk/solar/stereo/data.html STEREO/COR2 and SoHO/LASCO: https://sdac.virtualsolar.org/cgi/

NSO/GONG: https://gong.nso.edu/data/magmap/

HELCATS: https://www.helcats-fp7.eu

ICMECAT: https://doi.org/10.6084/m9.figshare.6356420

\section{Model}

ELEvoHI is available at https://zenodo.org/record/3873420.

EAGEL is available at https://zenodo.org/record/4154458.

\section{Results}

The visualization of each prediction result, i.e., movies and figures, can be downloaded from https://doi. org/10.6084/m9.figshare.12758312. 


\section{Software}

IDL ${ }^{\mathrm{TM}}$ Version 8.4

Python 3.7.6

SATPLOT: https://hesperia.gsfc.nasa.gov/ssw/stereo/secchi/idl/jpl/satplot/SATPLOT_Guide.pdf

Acknowledgments

T. Amerstorfer, J. Hinterreiter, M. Bauer, M. A. Reiss, C. Möstl, A. J. Weiss, R. L. Bailey, and U. V. Amerstorfer thank the Austrian Science Fund (FWF): P31265-N27, J4160-N27, P31659-N27, P31521-N27.

\section{References}

Altschuler, M. D., \& Newkirk, G. (1969). Magnetic fields and the structure of the solar corona. I: Methods of calculating coronal fields. Solar Physics, 9, 131-149. https://doi.org/10.1007/BF00145734

Amerstorfer, T., Hinterreiter, J., Reiss, M. A., Möstl, C., Davies, J. A., Bailey, R. L., et al. (2021). Evaluation of CME Arrival Prediction Using Ensemble Modeling Based on Heliospheric Imaging Observations. Space Weather, 19(1). http://dx.doi.org/10.1029/2020sw002553

Amerstorfer, T., Möstl, C., Hess, P., Temmer, M., Mays, M. L., Reiss, M. A., et al. (2018). Ensemble prediction of a Halo coronal mass ejection using heliospheric imagers. Space Weather, 16, 784-801. https://doi.org/10.1029/2017SW001786

Arge, C. N., Odstrcil, D., Pizzo, V. J., \& Mayer, L. R. (2003). Improved method for specifying solar wind speed near the Sun. In M. Velli, R. Bruno, F. Malara, \& B. Bucci (Eds.), Solar wind ten, American Institute of Physics Conference Series (Vol. 679, pp. 190-193). Pisa, Italy. https://doi.org/10.1063/1.1618574

Barnard, L., Owens, M. J., Scott, C. J., \& de Koning, C. A. (2020). Ensemble CME modeling constrained by heliospheric imager observations. AGU Advances, 1, e2020AV000214. https://doi.org/10.1029/2020AV000214

Barnard, L. A., de Koning, C. A., Scott, C. J., Owens, M. J., Wilkinson, J., \& Davies, J. A. (2017). Testing the current paradigm for space weather prediction with heliospheric imagers. Space Weather, 15, 782-803. https://doi.org/10.1002/2017SW001609

Barnard, L. A., Owens, M. J., Scott, C. J., \& Jones, S. R. (2019). Extracting inner-heliosphere solar wind speed information from heliospheric imager observations. Space Weather, 17, 925-938. https://doi.org/10.1029/2019SW002226

Barnes, D., Davies, J. A., Harrison, R. A., Byrne, J. P., Perry, C. H., Bothmer, V., et al. (2020). CMEs in the heliosphere: III. A statistical analysis of the kinematic properties derived from stereoscopic geometrical modelling techniques applied to CMEs detected in the heliosphere from 2008 to 2014 by STEREO/HI-1. arXiv:2006.14879

Braga, C. R., Vourlidas, A., Stenborg, G., Dal Lago, A., de Mendonça, R. R. S., \& Echer, E. (2020). Predicting the time of arrival of coronal mass ejections at earth from heliospheric imaging observations. Journal of Geophysical Research: Space Physics, 125, e27885. https:// doi.org/10.1029/2020JA027885

Brueckner, G. E., Howard, R. A., Koomen, M. J., Korendyke, C. M., Michels, D. J., Moses, J. D., et al. (1995). The large angle spectroscopic coronagraph (LASCO). Solar Physics, 162(1-2), 357-402. https://doi.org/10.1007/BF00733434

Cannon, P. S. (2013). Extreme space weather-A report published by the UK Royal Academy of Engineering. Space Weather, 11, 138-139. https://doi.org/10.1002/swe.20032

Chi, Y., Scott, C., Shen, C., Owens, M., Lang, M., Xu, M., et al. (2020). Using the "ghost front" to predict the arrival time and speed of CMEs at Venus and earth. The Astrophysical Journal, 899(2), 143. https://doi.org/10.3847/1538-4357/aba95a

Davies, J. A., Harrison, R. A., Perry, C. H., Möstl, C., Lugaz, N., Rollett, T., et al. (2012). A self-similar expansion model for use in solar wind transient propagation studies. The Astrophysical Journal, 750(1), 23. https://doi.org/10.1088/0004-637X/750/1/23

Davies, J. A., Harrison, R. A., Rouillard, A. P., Sheeley, N. R., Perry, C. H., Bewsher, D., et al. (2009). A synoptic view of solar transient evolution in the inner heliosphere using the heliospheric imagers on STEREO. Geophysical Research Letters, 36, L02102. https://doi. org/10.1029/2008GL036182

Davies, J. A., Perry, C. H., Trines, R. M. G. M., Harrison, R. A., Lugaz, N., Möstl, C., et al. (2013). Establishing a stereoscopic technique for determining the kinematic properties of solar wind Transients based on a generalized self-similarly expanding circular geometry. The Astrophysical Journal, 777(2), 167. https://doi.org/10.1088/0004-637X/777/2/167

Dumbović, M., Čalogović, J., Vršnak, B., Temmer, M., Mays, M. L., Veronig, A., \& Piantschitsch, I. (2018). The drag-based ensemble model (DBEM) for coronal mass ejection propagation. The Astrophysical Journal, 854(2), 180. https://doi.org/10.3847/1538-4357/aaaa66

Eyles, C. J., Harrison, R. A., Davis, C. J., Waltham, N. R., Shaughnessy, B. M., Mapson-Menard, H. C. A., et al. (2009). The heliospheric imagers onboard the STEREO mission. Solar Physics, 254(2), 387-445. https://doi.org/10.1007/s11207-008-9299-0

Gopalswamy, N., Lara, A., Lepping, R. P., Kaiser, M. L., Berdichevsky, D., \& Cyr, S. O. C. (2000). Interplanetary acceleration of coronal mass ejections. Geophysical Research Letters, 27(2), 145-148. https://doi.org/10.1029/1999GL003639

Gosling, J. T., Bame, S. J., McComas, D. J., \& Phillips, J. L. (1990). Coronal mass ejections and large geomagnetic storms. Geophysical Research Letters, 17(7), 901-904. https://doi.org/10.1029/GL017i007p00901

Harrison, R. A., Davies, J. A., Barnes, D., Byrne, J. P., Perry, C. H., Bothmer, V., et al. (2018). CMEs in the heliosphere: I. A statistical analysis of the observational properties of CMEs detected in the heliosphere from 2007 to 2017 by STEREO/HI-1. Solar Physics, 293(5), 77. https://doi.org/10.1007/s11207-018-1297-2

Howard, R. A., Moses, J. D., Vourlidas, A., Newmark, J. S., Socker, D. G., Plunkett, S. P., et al. (2008). Sun earth connection coronal and heliospheric investigation (SECCHI). Space Science Reviews, 136(1-4), 67-115. https://doi.org/10.1007/s11214-008-9341-4

Hundhausen, A. J., Stanger, A. L., \& Serbicki, S. A. (1994). Mass and energy contents of coronal mass ejections: SMM results from 1980 and 1984-1988. In J. J. Hunt (Ed.), Solar dynamic phenomena and solar wind consequences, the third SoHO workshop (Vol. 373, pp. 409). Estes Park, CO: ESA Special Publication.

Kaiser, M. L., Kucera, T. A., Davila, J. M., Cyr, S. O. C., Guhathakurta, M., \& Christian, E. (2008). The STEREO mission: An introduction. Space Science Reviews, 136(1-4), 5-16. https://doi.org/10.1007/s11214-007-9277-0

Kay, C., Mays, M. L., \& Verbeke, C. (2020). Identifying critical input parameters for improving drag-based CME arrival time predictions. Space Weather, 18, e02382. https://doi.org/10.1029/2019SW002382

Kay, C., \& Nieves-Chinchilla, T. (2020). Modeling interplanetary expansion and deformation of CMEs with ANTEATR-PARADE I: Relative contribution of different forces. arXiv:2011.06030

Kay, C., \& Opher, M. (2015). The heliocentric distance where the deflections and rotations of solar coronal mass ejections occur. The Astrophysical Journal Letters, 811(2), L36. https://doi.org/10.1088/2041-8205/811/2/L36

Kilpua, E. K. J., Jian, L. K., Li, Y., Luhmann, J. G., \& Russell, C. T. (2012). Observations of ICMEs and ICME-like solar wind structures from 2007-2010 using near-earth and STEREO observations. Solar Physics, 281(1), 391-409. https://doi.org/10.1007/s11207-012-9957-0 
Kraft, S., Puschmann, K. G., \& Luntama, J. P. (2017). Remote sensing optical instrumentation for enhanced space weather monitoring from the L1 and L5 Lagrange points. In B. Cugny, N. Karafolas, \& Z. Sodnik (Eds.), International conference on space optics "ICSO 2016" (Vol. 10562, pp. 115-123). SPIE. https://doi.org/10.1117/12.2296100

Kubicka, M., Möstl, C., Amerstorfer, T., Boakes, P. D., Feng, L., Eastwood, J. P., \& Törmänen, O. (2016). Prediction of geomagnetic storm strength from inner heliospheric in situ observations. The Astrophysical Journal, 833(2), 255. https://doi.org/10.3847/1538-4357/833/2/255

Lavraud, B., Liu, Y., Segura, K., He, J., Qin, G., Temmer, M., et al. (2016). A small mission concept to the Sun-Earth Lagrangian L5 point for innovative solar, heliospheric and space weather science. Journal of Atmospheric and Solar-Terrestrial Physics, 146, 171-185. https:// doi.org/10.1016/j.jastp.2016.06.004

Liu, Y., Davies, J. A., Luhmann, J. G., Vourlidas, A., Bale, S. D., \& Lin, R. P. (2010). Geometric triangulation of imaging observations to track coronal mass ejections continuously out to 1 AU. The Astrophysical Journal Letters, 710(1), L82-L87. https://doi. org/10.1088/2041-8205/710/1/L82

Liu, Y. D., Luhmann, J. G., Kajdič, P., Kilpua, E. K. J., Lugaz, N., Nitta, N. V., et al. (2014). Observations of an extreme storm in interplanetary space caused by successive coronal mass ejections. Nature Communications, 5, 3481. https://doi.org/10.1038/ncomms4481

Lugaz, N. (2010). Accuracy and limitations of fitting and stereoscopic methods to determine the direction of coronal mass ejections from heliospheric imagers observations. Solar Physics, 267(2), 411-429. https://doi.org/10.1007/s11207-010-9654-9

Lugaz, N., Farrugia, C. J., Davies, J. A., Möstl, C., Davis, C. J., Roussev, I. I., \& Temmer, M. (2012). The deflection of the two interacting coronal mass ejections of 2010 may 23-24 as revealed by combined in situ measurements and heliospheric imaging. The Astrophysical Journal, 759(1), 68. https://doi.org/10.1088/0004-637X/759/1/68

Lugaz, N., Hernandez-Charpak, J. N., Roussev, I. I., Davis, C. J., Vourlidas, A., \& Davies, J. A. (2010). Determining the azimuthal properties of coronal mass ejections from multi-spacecraft remote-sensing observations with STEREO SECCHI. The Astrophysical Journal, 715(1), 493-499. https://doi.org/10.1088/0004-637X/715/1/493

Luhmann, J. G., Curtis, D. W., Schroeder, P., McCauley, J., Lin, R. P., Larson, D. E., et al. (2008). STEREO IMPACT investigation goals, measurements, and data products overview. Space Science Reviews, 136(1-4), 117-184. https://doi.org/10.1007/s11214-007-9170-X

Manoharan, P. K., Gopalswamy, N., Yashiro, S., Lara, A., Michalek, G., \& Howard, R. A. (2004). Influence of coronal mass ejection interaction on propagation of interplanetary shocks. Journal of Geophysical Research, 109, A06109. https://doi.org/10.1029/2003JA010300

Manoharan, P. K., \& Mujiber Rahman, A. (2011). Coronal mass ejections-Propagation time and associated internal energy. Journal of Atmospheric and Solar-Terrestrial Physics, 73(5-6), 671-677. https://doi.org/10.1016/j.jastp.2011.01.017

Mierla, M., Inhester, B., Antunes, A., Boursier, Y., Byrne, J. P., Colaninno, R., et al. (2010). On the 3-D reconstruction of coronal mass ejections using coronagraph data. Annales Geophysicae, 28(1), 203-215. https://doi.org/10.5194/angeo-28-203-2010

Möstl, C., \& Davies, J. A. (2013). Speeds and arrival times of solar transients approximated by self-similar expanding circular fronts. Solar Physics, 285(1-2), 411-423. https://doi.org/10.1007/s11207-012-9978-8

Möstl, C., Isavnin, A., Boakes, P. D., Kilpua, E. K. J., Davies, J. A., Harrison, R. A., et al. (2017). Modeling observations of solar coronal mass ejections with heliospheric imagers verified with the Heliophysics System Observatory. Space Weather, 15, 955-970. https://doi. org/10.1002/2017SW001614

Möstl, C., Rollett, T., Frahm, R. A., Liu, Y. D., Long, D. M., Colaninno, R. C., et al. (2015). Strong coronal channelling and interplanetary evolution of a solar storm up to Earth and Mars. Nature Communications, 6, 7135. https://doi.org/10.1038/ncomms8135

Möstl, C., Rollett, T., Lugaz, N., Farrugia, C. J., Davies, J. A., Temmer, M., et al. (2011). Arrival time calculation for interplanetary coronal mass ejections with circular fronts and application to STEREO observations of the 2009 February 13 eruption. The Astrophysical Journal, 741(1), 34. https://doi.org/10.1088/0004-637X/741/1/34

Möstl, C., Weiss, A. J., Bailey, R. L., Reiss, M. A., Amerstorfer, U. V., Amerstorfer, T., et al. (2020). Prediction of the in situ coronal mass ejection rate for solar cycle 25: Implications for Parker Solar Probe in situ observations. arXiv:2007.14743

Nieves-Chinchilla, T., Vourlidas, A., Raymond, J. C., Linton, M. G., Al-haddad, N., Savani, N. P., et al. (2018). Understanding the internal magnetic field configurations of ICMEs using more than 20 Years of wind observations. Solar Physics, 293(2), 25. https://doi. org/10.1007/s11207-018-1247-z

Odstrcil, D., Pizzo, V. J., Linker, J. A., Riley, P., Lionello, R., \& Mikic, Z. (2004). Initial coupling of coronal and heliospheric numerical magnetohydrodynamic codes. Journal of Atmospheric and Solar-Terrestrial Physics, 66(15-16), 1311-1320. https://doi.org/10.1016/j. jastp.2004.04.007

Owens, M. J., Lockwood, M., \& Barnard, L. A. (2017). Coronal mass ejections are not coherent magnetohydrodynamic structures. Scientific Reports, 7, 4152. https://doi.org/10.1038/s41598-017-04546-3

Owens, M. J., Lockwood, M., \& Barnard, L. A. (2020). The value of CME arrival time forecasts for space weather mitigation. Space Weather, 18, e2020SW002507. https://doi.org/10.1029/2020SW002507

Owens, M. J., \& Riley, P. (2017). Probabilistic solar wind forecasting using large ensembles of near-Sun conditions with a simple one-dimensional "upwind" scheme. Space Weather, 15, 1461-1474. https://doi.org/10.1002/2017SW001679

Paouris, E., \& Mavromichalaki, H. (2017). Effective acceleration model for the arrival time of interplanetary shocks driven by coronal mass ejections. Solar Physics, 292(12), 180. https://doi.org/10.1007/s11207-017-1212-2

Pomoell, J., \& Poedts, S. (2018). Euhforia: European heliospheric forecasting information asset. Journal of Space Weather and Space Climate, 8, A35. https://doi.org/10.1051/swsc/2018020

Reiss, M. A., MacNeice, P. J., Mays, L. M., Arge, C. N., Möstl, C., Nikolic, L., \& Amerstorfer, T. (2019). Forecasting the ambient solar wind with numerical models. I. On the implementation of an operational framework. The Astrophysical Journal Supplement Series, 240,35 https://doi.org/10.3847/1538-4365/aaf8b3

Reiss, M. A., MacNeice, P. J., Muglach, K., Arge, C. N., Möstl, C., Riley, P., et al. (2020). Forecasting the ambient solar wind with numerical models. II. An adaptive prediction system for specifying solar wind speed near the Sun. The Astrophysical Journal, 891(2), 165. https:// doi.org/10.3847/1538-4357/ab78a0

Richardson, I. G., \& Cane, H. V. (2010). Near-Earth interplanetary coronal mass ejections during solar cycle 23 (1996-2009): Catalog and summary of properties. Solar Physics, 264(1), 189-237. https://doi.org/10.1007/s11207-010-9568-6

Richardson, I. G., \& Cane, H. V. (2012). Near-earth solar wind flows and related geomagnetic activity during more than four solar cycles (1963-2011). Journal of Space Weather and Space Climate, 2, A02. https://doi.org/10.1051/swsc/2012003

Riley, P., \& Crooker, N. U. (2004). Kinematic treatment of coronal mass ejection evolution in the solar wind. The Astrophysical Journal, 600(2), 1035-1042. https://doi.org/10.1086/379974

Riley, P., \& Lionello, R. (2011). Mapping solar wind streams from the Sun to 1 AU: A comparison of techniques. Solar Physics, $270,575-592$. https://doi.org/10.1007/s11207-011-9766-x 
Riley, P., Mays, M. L., Andries, J., Amerstorfer, T., Biesecker, D., Delouille, V., et al. (2018). Forecasting the arrival time of coronal mass ejections: Analysis of the CCMC CME scoreboard. Space Weather, 16, 1245-1260. https://doi.org/10.1029/2018SW001962

Rollett, T., Möstl, C., Isavnin, A., Davies, J. A., Kubicka, M., Amerstorfer, U. V., \& Harrison, R. A. (2016). ElEvoHI: A novel CME prediction tool for heliospheric imaging combining an elliptical front with drag-based model fitting. The Astrophysical Journal, 824(2), 131. https:// doi.org/10.3847/0004-637X/824/2/131

Rollett, T., Möstl, C., Temmer, M., Frahm, R. A., Davies, J. A., Veronig, A. M., et al. (2014). Combined multipoint remote and in situ observations of the asymmetric evolution of a fast solar coronal mass ejection. The Astrophysical Journal Letters, 790(1), L6. https://doi. org/10.1088/2041-8205/790/1/L6

Rouillard, A. P., Davies, J. A., Forsyth, R. J., Rees, A., Davis, C. J., Harrison, R. A., et al. (2008). First imaging of corotating interaction regions using the STEREO spacecraft. Geophysical Research Letters, 35, L10110. https://doi.org/10.1029/2008GL033767

Ruffenach, A., Lavraud, B., Farrugia, C. J., Démoulin, P., Dasso, S., Owens, M. J., et al. (2015). Statistical study of magnetic cloud erosion by magnetic reconnection. Journal of Geophysical Research: Space Physics, 120, 43-60. https://doi.org/10.1002/2014JA020628

Savani, N. P., Owens, M. J., Rouillard, A. P., Forsyth, R. J., \& Davies, J. A. (2010). Observational evidence of a coronal mass ejection distortion directly attributable to a structured solar wind. The Astrophysical Journal Letters, 714(1), L128-L132. https://doi. org/10.1088/2041-8205/714/1/L128

Schatten, K. H. (1971). Current sheet magnetic model for the solar corona. Cosmic Electrodynamics, 2, 232-245.

Schatten, K. H., Wilcox, J. M., \& Ness, N. F. (1969). A model of interplanetary and coronal magnetic fields. Solar Physics, 6, $442-455$. https://doi.org/10.1007/BF00146478

Scott, C. J., Owens, M. J., de Koning, C. A., Barnard, L. A., Jones, S. R., \& Wilkinson, J. (2019). Using ghost fronts within STEREO heliospheric imager data to infer the evolution in longitudinal structure of a coronal mass ejection. Space Weather, 17, 539-552. https://doi. org/10.1029/2018SW002093

Sheeley, N. R., Walters, J. H., Wang, Y. M., \& Howard, R. A. (1999). Continuous tracking of coronal outflows: Two kinds of coronal mass ejections. Journal of Geophysical Research, 104(A11), 24739-24768. https://doi.org/10.1029/1999JA900308

Srivastava, N., \& Venkatakrishnan, P. (2004). Solar and interplanetary sources of major geomagnetic storms during 1996-2002. Journal of Geophysical Research, 109, A10103. https://doi.org/10.1029/2003JA010175

Temmer, M., \& Nitta, N. V. (2015). Interplanetary propagation behavior of the fast coronal mass ejection on 23 July 2012 . Solar Physics, 290(3), 919-932. https://doi.org/10.1007/s11207-014-0642-3

Thernisien, A., Vourlidas, A., \& Howard, R. A. (2009). Forward modeling of coronal mass ejections using STEREO/SECCHI data. Solar Physics, 256(1-2), 111-130. https://doi.org/10.1007/s11207-009-9346-5

Thernisien, A. F. R., Howard, R. A., \& Vourlidas, A. (2006). Modeling of flux rope coronal mass ejections. The Astrophysical Journal, 652(1), 763-773. https://doi.org/10.1086/508254

Volpes, L., \& Bothmer, V. (2015). An application of the stereoscopic self-similar-expansion model to the determination of CME-driven shock parameters. Solar Physics, 290(10), 3005-3022. https://doi.org/10.1007/s11207-015-0775-Z

Vršnak, B., Žic, T., Vrbanec, D., Temmer, M., Rollett, T., Möstl, C., et al. (2013). Propagation of interplanetary coronal mass ejections: The drag-based model. Solar Physics, 285(1-2), 295-315. https://doi.org/10.1007/s11207-012-0035-4

Wang, Y., Shen, C., Wang, S., \& Ye, P. (2004). Deflection of coronal mass ejection in the interplanetary medium. Solar Physics, 222(2), 329-343. https://doi.org/10.1023/B:SOLA.0000043576.21942.aa

Wang, Y., Wang, B., Shen, C., Shen, F., \& Lugaz, N. (2014). Deflected propagation of a coronal mass ejection from the corona to interplanetary space. Journal of Geophysical Research: Space Physics, 119, 5117-5132. https://doi.org/10.1002/2013JA019537

Wang, Y.-M., \& Sheeley, N. R. Jr. (1995). Solar implications of ULYSSES interplanetary field measurements. The Astrophysical Journal Letters, 447, L143. https://doi.org/10.1086/309578

Žic, T., Vršnak, B., \& Temmer, M. (2015). Heliospheric propagation of coronal mass ejections: Drag-based model fitting. The Astrophysical Journal Supplement Series, 218(2), 32. https://doi.org/10.1088/0067-0049/218/2/32

Zuccarello, F. P., Bemporad, A., Jacobs, C., Mierla, M., Poedts, S., \& Zuccarello, F. (2012). The role of streamers in the deflection of coronal mass ejections: Comparison between STEREO three-dimensional reconstructions and numerical simulations. The Astrophysical Journal, 744(1), 66. https://doi.org/10.1088/0004-637X/744/1/66 